\title{
Krüppel-like factor 4 is a negative regulator of STAT3-induced glomerular epithelial cell proliferation
}

Chelsea C. Estrada, ${ }^{1}$ Praharshasai Paladugu, ${ }^{1}$ Yiqing Guo, ${ }^{1}$ Jesse Pace, ${ }^{1}$ Monica P. Revelo, ${ }^{2}$ David J. Salant, ${ }^{3}$ Stuart J. Shankland, ${ }^{4}$ Vivette D. D'Agati, ${ }^{5}$ Anita Mehrotra, ${ }^{6,7}$ Stephanie Cardona, ${ }^{1}$ Agnieszka B. Bialkowska, ${ }^{8}$ Vincent W. Yang, ${ }^{8}$ John C. He, ${ }^{6,7}$ and Sandeep K. Mallipattu ${ }^{1,9}$

'Division of Nephrology, Department of Medicine, Stony Brook University, Stony Brook, New York, USA. 'Department of Pathology, University of Utah, Salt Lake City, Utah, USA. ${ }^{3}$ Division of Nephrology, Department of Medicine, Boston University School of Medicine, Boston, Massachusetts, USA. ${ }^{4}$ Division of Nephrology, Department of Medicine, University of Washington School of Medicine, Seattle, Washington, USA. ${ }^{5}$ Department of Pathology, Columbia University, New York, New York, USA. ${ }^{6}$ Division of Nephrology, Department of Medicine, Icahn School of Medicine at Mount Sinai, New York, New York, USA. ${ }^{7}$ Renal Section, James J. Peters VA Medical Center, New York, New York, USA. ${ }^{8}$ Division of Gastroenterology, Department of Medicine, Stony Brook University, Stony Brook, New York, USA. ${ }^{9}$ Renal Section, Northport VA Medical Center, Northport, New York, USA.

Pathologic glomerular epithelial cell (CEC) hyperplasia is characteristic of both rapidly progressive glomerulonephritis (RPCN) and subtypes of focal segmental glomerulosclerosis (FSCS). Although initial podocyte injury resulting in activation of STAT3 signals CEC proliferation in both diseases, mechanisms regulating this are unknown. Here, we show that the loss of Krüppel-like factor 4 (KLF4), a zinc-finger transcription factor, enhances GEC proliferation in both RPGN and FSCS due to dysregulated STAT3 signaling. We observed that podocyte-specific knockdown of KIf4 (C57BL/6]) increased STAT3 signaling and exacerbated crescent formation after nephrotoxic serum treatment. Interestingly, podocyte-specific knockdown of $K I$ If 4 in the FVB/N background alone was sufficient to activate STAT3 signaling, resulting in FSCS with extracapillary proliferation, as well as renal failure and reduced survival. In cultured podocytes, loss of KLF4 resulted in STAT3 activation and cell-cycle reentry, leading to mitotic catastrophe. This triggered IL-6 release into the supernatant, which activated STAT3 signaling in parietal epithelial cells. Conversely, either restoration of KLF4 expression or inhibition of STAT3 signaling improved survival in KLF4-knockdown podocytes. Finally, human kidney biopsy specimens with RPGN exhibited reduced KLF4 expression with a concomitant increase in phospho-STAT3 expression as compared with controls. Collectively, these results suggest the essential role of KLF4/STAT3 signaling in podocyte injury and its regulation of aberrant CEC proliferation.

Conflict of interest: The authors have declared that no conflict of interest exists.

Submitted: October 20, 2017 Accepted: May 14, 2018 Published: June 21, 2018

\section{Reference information:} JCI Insight. 2018;3(12):e98214. https://doi.org/10.1172/jici. insight.98214.

\section{Introduction}

Proliferation of glomerular epithelial cells (GECs) is the dominant histologic feature of rapidly progressive glomerulonephritis (RPGN), as well as subtypes of focal segmental glomerulosclerosis (FSGS), particularly the collapsing and cellular variants $(1,2)$. Lineage tracing experiments have shown that the majority of proliferating epithelial cells in the hyperplastic lesions in RPGN and collapsing FSGS are parietal in origin (2), with a lessor contribution from podocytes (3). This pathologic GEC proliferation in RPGN is the main component of the crescentic lesion, which also contains inflammatory cells, capillary injury, and/or necrosis (4). Similarly, in collapsing FSGS, these proliferative lesions are described as pseudo-crescents, which refers to segmental or global collapse of the capillary tuft with pronounced epithelial cell hyperplasia (4). Furthermore, crosstalk between podocytes and parietal epithelial cells (PECs) is likely to have a role in the pathogenesis of these hyperplastic lesions, as podocyte-specific insult has been demonstrated to incite crescentic lesions (5).

Activation of signal transducer and activator of transcription 3 (STAT3) has been implicated in the initiation and progression of both RPGN and collapsing FSGS (6, 7). Podocyte-specific deletion of Stat3 
ameliorated GEC proliferation and crescent formation in a murine model of RPGN, nephrotoxic serum (NTS) nephritis (6). In addition, global reduction of Stat3 reduced hyperplastic glomerular lesions in a murine model of HIV-associated nephropathy (HIVAN), associated with collapsing FSGS (7). These proproliferative and proinflammatory functions of STAT3 have also been well described in nonrenal diseases, where inhibition of STAT3 signaling has been targeted as a therapeutic in malignancies and autoimmune disorders $(8,9)$. Although activation of STAT3 signaling plays an important role in the pathogenesis of RPGN and subtypes of FSGS, the regulation of STAT3 signaling in the podocyte remains unclear.

Krüppel-like factors (KLFs) are a family of zinc-finger transcription factors that exert diverse effects on key biologic processes, including tumorigenesis, immune cell activation, and pluripotency $(10,11)$. Although several members in the family have been implicated in cell differentiation (11-13), KLF4 was first identified as a negative regulator of proliferation by inducing cell-cycle arrest and restoring prodifferentiation markers in intestinal epithelial cells $(10,14)$. KLF4 has been reported to inhibit cell-cycle progression by various mechanisms, including transcriptional activation of the cyclin-dependent kinase inhibitors (CDKIs) $C D K N 1 A / p 27$ and $C D K N 1 C / p 57(15,16)$, as well as direct inhibition of $C C N E 1(17)$ and $C C N B 1$ (18), following DNA damage. In this regard, KLF4 has been shown to possess both prodifferentiation and antiproliferative properties in several organs and cell types, such as the kidney (19), the intestine (20), and the neuron (21). Apart from its critical role in cell-cycle regulation, KLF4 was recently reported to inhibit activation of STAT3, where genetic deletion of Klf4 increased STAT3 signaling and enhanced axon regeneration in retinal ganglion cells after optic nerve injury and in neurons after traumatic brain injury $(21,22)$. In addition, KLF4 was shown to be a direct downstream target of STAT3 signaling in embryonic stem cells $(23,24)$, suggesting a negative-feedback mechanism.

In the kidney, KLF4 has been studied in endothelial cells, where it plays a protective role in acute kidney injury (25), as well as in podocytes, where podocyte-specific knockdown of Klf4 increased the susceptibility to podocyte injury in proteinuric murine models $(11,19,26)$. Furthermore, these authors also demonstrated that KLF4 might mediate the epigenetic changes in podocytes induced by angiotensin receptor blockers (26). However, the role of podocyte-specific KLF4 in diseases of aberrant GEC proliferation has not been described.

Based on these findings, we sought to determine the role of podocyte-specific KLF4 in regulating STAT3 signaling in murine models of proliferative glomerulonephritis and how initial glomerular and/or podocyte injury might lead to extracapillary hypercellularity involving PEC proliferation. Here, we initially demonstrated that the podocyte-specific loss of Klf4 (C57BL/6J background) exacerbated GEC proliferation and renal insufficiency in the NTS nephritis murine model. We also observed that the podocyte-specific loss of Klf4 on the FVB/N background renders the activation of glomerular STAT3 signaling, leading to FSGS with GEC proliferation and renal failure, with significant reduced overall survival. Furthermore, we demonstrated that KLF4 is critical to the maintenance of mature podocyte differentiation markers by preventing STAT3 activation and cell cycle reentry. Finally, we show that the activation of STAT3 signaling inversely correlated with KLF4 expression in the glomeruli of kidney biopsies with RPGN as compared with control specimens.

\section{Results}

Podocyte-specific loss of Klf4 exacerbates crescent formation and renal dysfunction after NTS treatment. Earlier studies have reported that the podocyte-specific loss of Klf4 increases the susceptibility to podocyte injury in proteinuric murine models $(19,26)$. In addition, KLF4 was initially described to play an important role in intestinal epithelial cell differentiation (14). Since podocytes are terminally differentiated, postmitotic cells and express KLF4 in the unperturbed state (19), we sought to determine whether the loss of Klf4 specifically in the podocytes exacerbates glomerular diseases with aberrant GEC proliferation. To test this hypothesis, we generated mice with podocyte-specific Klf4 knockdown using the Cre-loxP recombination system. Podocin-Cre mice (C57BL/6J) were crossed with Klf4l/fl $(\mathrm{C} 57 \mathrm{BL} / 6 \mathrm{~J})$ to generate Podocin-Cre Klf4l/fl mice (F2 generation). To demonstrate Klf4 knockdown in podocytes, we isolated primary GECs from Podocin-Cre Klffl/fl and PodocinCre $\mathrm{Klf4}^{+/+}$mice and observed a significant reduction in KLF4 mRNA and protein expression in Podocin-Cre Klffl/fl mice as compared with Podocin-Cre Klf4 ${ }^{+/+}$mice (Supplemental Figure 1, A and B; supplemental material available online with this article; https://doi.org/10.1172/jci.insight.98214DS1). Podocin-Cre Klf4//fl mice were fertile with no significant differences in albuminuria, serum creatinine, or body weight as compared with Podocin-Cre $\mathrm{Klf4}^{+/+}$mice at 10 weeks of age on the C57BL/6J background. 
A
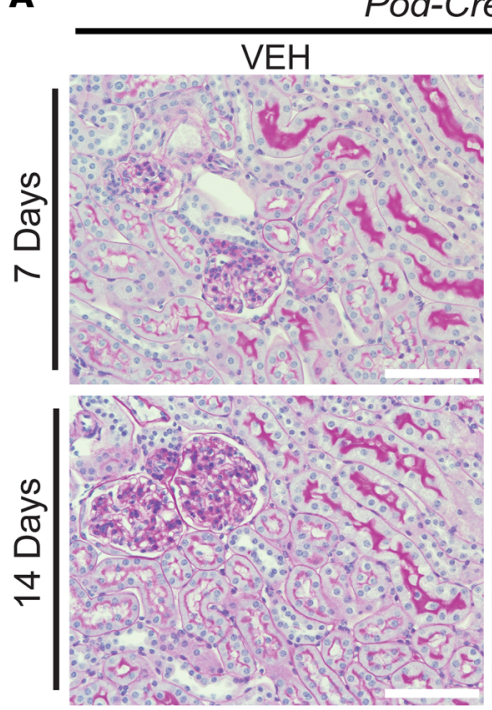

Pod-Cre KIf4+/+
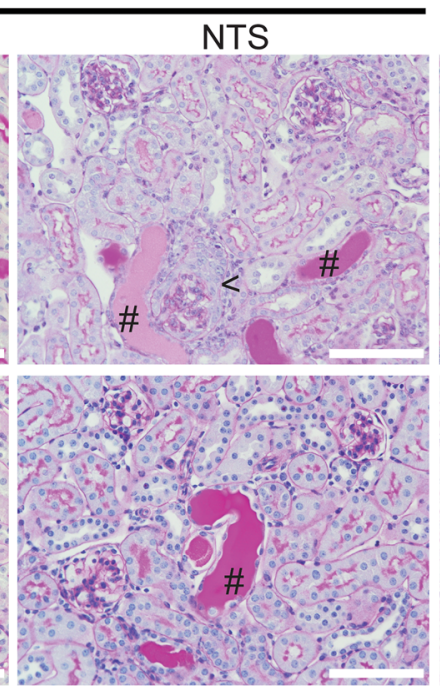

Pod-Cre KIf $4^{f / f I}$
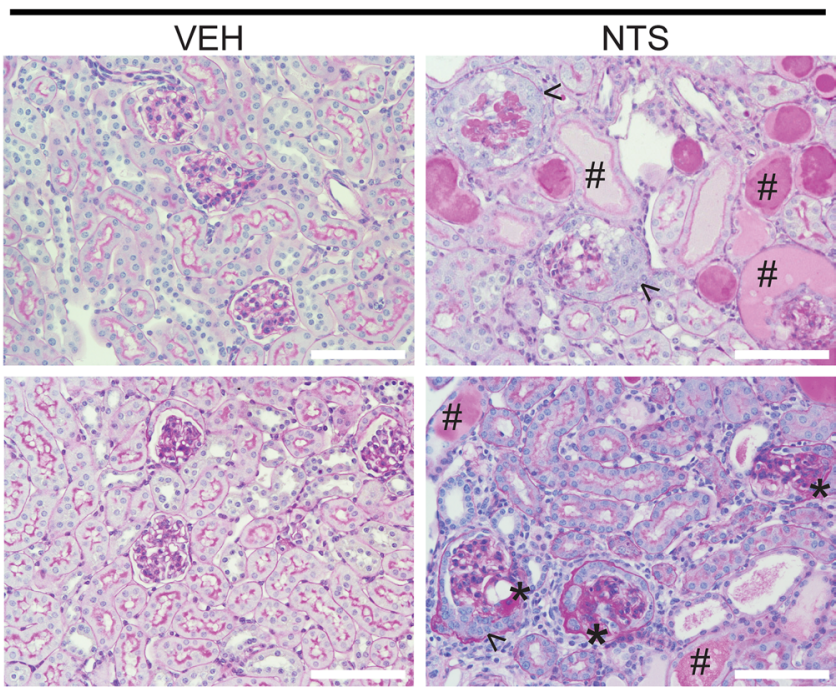

B

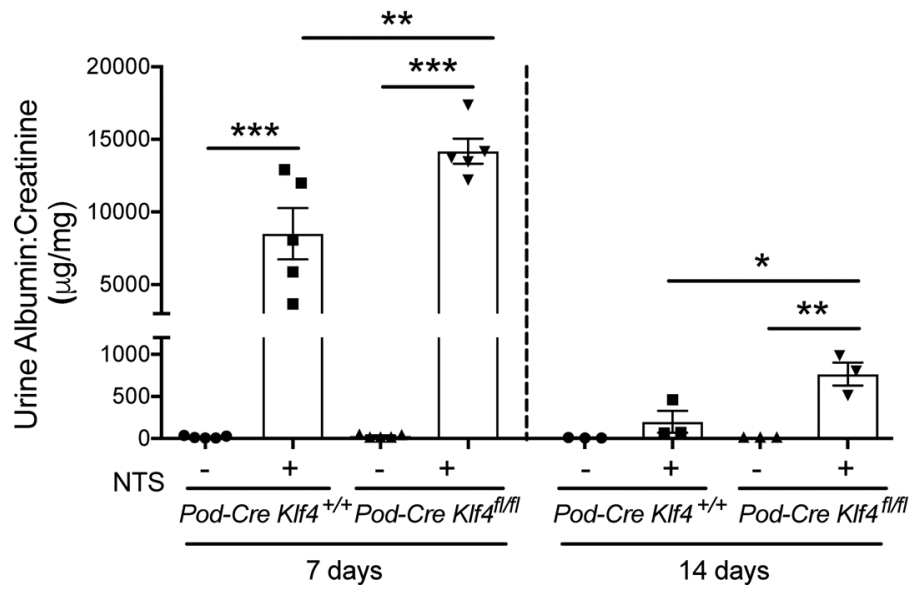

C

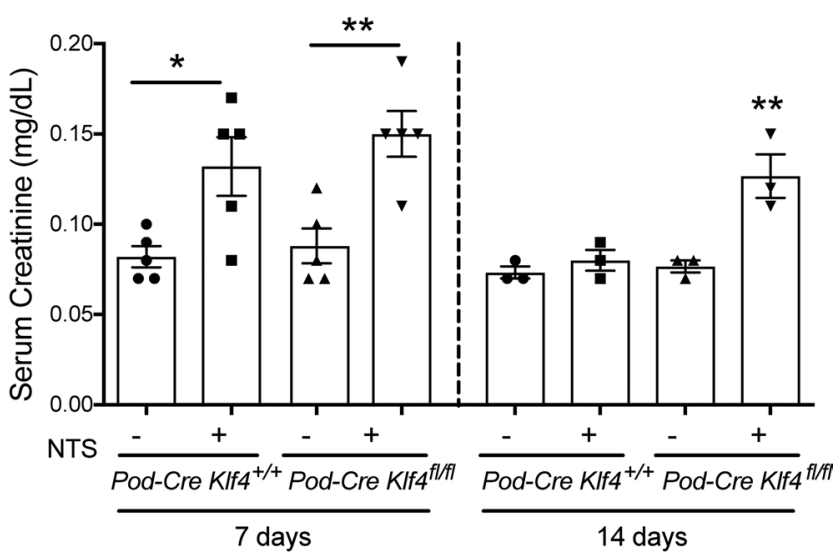

Figure 1. NTS-treated Podocin-Cre KIf $4^{f / f l}$ mice exhibit a significant increase in crescent formation, albuminuria, and renal failure. Podocin-Cre KIf $4^{f / f l}$ and Podocin-Cre KIf $4^{+/+}$mice were treated with nephrotoxic serum (NTS) or VEH for 7 and 14 days. (A) Representative images of periodic acid-Schiff (PAS) staining $(\times 20)$ ( $n=5$ in each group at 7 days and $n=3$ in each group at 14 days). Arrowheads show crescents. Pound signs mark tubular casts and dilation. Asterisks show sclerotic glomeruli. (B) Albuminuria (urine albumin/creatinine) and (C) serum creatinine at 7 and 14 days after treatment ( $n=5$ at 7 days, $n$ $=3$ at 14 days; ${ }^{*} P<0.05,{ }^{* *} P<0.01,{ }^{* * *} P<0.001$; Kruskal-Wallis test with Dunn's post hoc test). Scale bars: $100 \mu \mathrm{m}$.

To assess whether the podocyte-specific loss of Klf4 increases the susceptibility to podocyte injury and GEC proliferation, we utilized an accelerated autologous-phase NTS model, using i.p. sheep NTS in mice preimmunized with sheep IgG to generate crescentic glomerulonephritis $(6,27)$. Podocin-Cre Klf4 $4^{+/+}$and Podocin-Cre Klf4 ${ }^{f l f l}$ mice were treated with NTS or vehicle (VEH). Seven days after treatment, NTS-treated Podocin-Cre Klf4l/fl mice exhibited a significant increase in the percentage of glomeruli with crescents as compared with all other groups (Figure 1A and Table 1). Although all NTS-treated mice exhibited a reduction in crescent formation by 14 days as compared with 7 days, we only observed a significant increase in the percent FSGS lesions and tubulointerstitial inflammation in the NTS-treated Podocin-Cre Klffl/fl as compared with VEH-treated groups (Figure 1A and Table 1). Functionally, we also observed a significant increase in

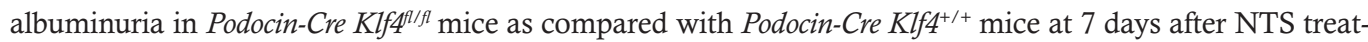
ment (Figure 1B). Interestingly, the albuminuria returned to baseline in the NTS-treated Podocin-Cre Klf4 ${ }^{+/+}$ mice as compared with NTS-treated Podocin-Cre Klf4l/fl mice by 14 days (Figure 1B). Similarly, serum creatinine was increased in all NTS-treated mice as compared with VEH-treated mice at 7 days, but all returned to baseline at 14 days - except for NTS-treated Podocin-Cre Klf4t/lfl (Figure 1C). Combined, these data suggest 
Table 1. Quantification of histologic change in nephrotoxic serum nephritis model

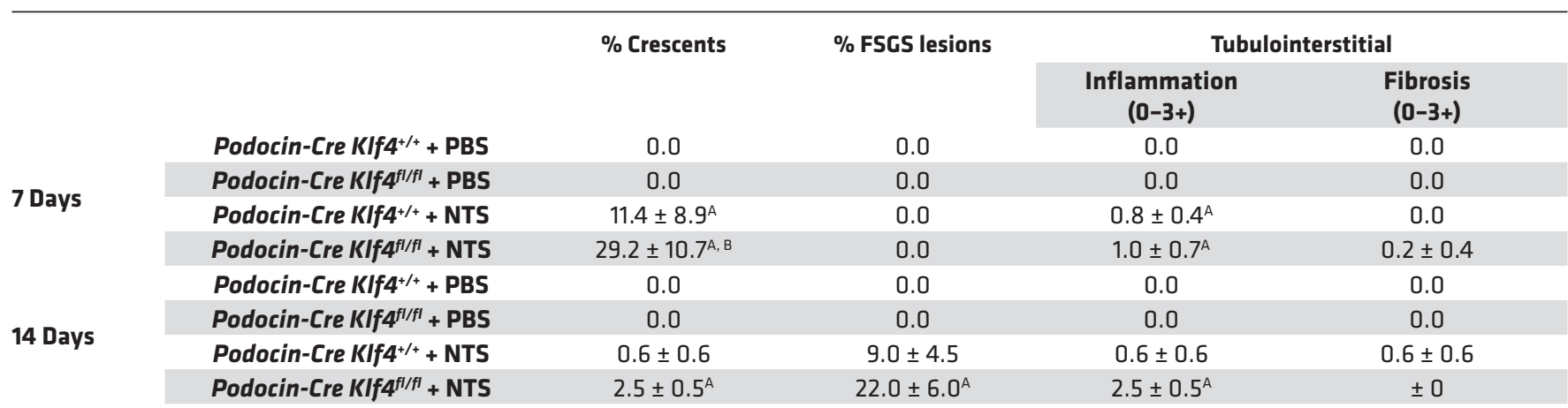

${ }^{A} P<0.05$ compared with PBS-treated mice. ${ }^{B} P<0.05$ compared with NTS-treated Podocin-Cre $\mathrm{KIf4}^{+/+}$mice. Tubulointerstitial scoring: 0 , none; $1,<10 \% ; 2$, $10 \%-25 \%$; $3,>25 \%-50 \%$.

that the conditional knockdown of Klf4 in podocytes exacerbates crescent formation and renal dysfunction, with eventual FSGS in a murine model of RPGN.

Podocyte-specific Klf4 knockdown exacerbates loss of podocyte markers after NTS treatment. Loss of mature podocyte differentiation markers has previously been reported in crescentic glomerulonephritis $(2,6)$. To ascertain whether podocyte-specific Klf4 knockdown exacerbates the loss of podocyte differentiation markers after NTS treatment, we evaluated the expression of Nephrin, Synaptopodin, and Wilms Tumor 1 (WT1) by immunostaining. While both NTS-treated groups exhibited a reduction in Nephrin, Synaptopodin, and WT1 expression as compared with VEH-treated controls, this was significantly exaggerated in NTS-treated Podocin-Cre Klf4/ ${ }^{\text {I/f }}$ mice as compared with all other groups at 7 days after treatment (Figure 2, A-D). Taken together, these data show that the podocyte-specific loss of Klf4 exacerbates the loss of mature podocyte markers in a murine model of RPGN.

NTS-treated podocyte-specific Klf4-knockdown mice exhibit increased STAT3 activation. Since podocyte-derived STAT3 signaling has been suggested to drive GEC proliferation and crescent formation after NTS treatment (6), we initially measured activation of STAT3 signaling by performing immunofluorescence for phospho-STAT3 (tyrosine 705; Y705) at 7 days after treatment. Although we did not observe any substan-

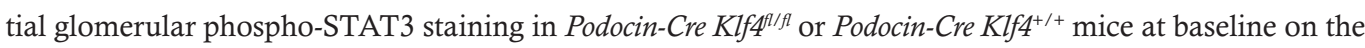
C57BL/6J background (Figure 3, A and B), NTS-treated Podocin-Cre Klf4//fl mice exhibited a significant increase in the percentage of glomeruli with positive nuclear staining for phospho-STAT3 compared with NTS-treated Podocin-Cre Klf4 ${ }^{+/+}$mice (Figure 3, A and B). We also observed a significant increase in mRNA expression of downstream targets of STAT3 signaling (Intercellular adhesion molecule 1 [Icam-1], Il-6, and Suppressor of cytokine signaling 3 [Socs3]) in isolated glomeruli from NTS-treated Podocin-Cre Klffl/lf mice as compared with all other groups, with no significant changes in Stat3 mRNA expression (Figure 3, C-F).

To determine whether the increased STAT3 signaling correlated with increased GEC proliferation, we performed immunostaining for the proliferation marker Ki67. We observed a significant increase in the number of $\mathrm{Ki} 7^{+}$cells per glomerular cross-sectional area in NTS-treated Podocin-Cre Klfl//fl mice as compared with all other groups (Figure 3G), with no significant glomerular Ki67 staining in VEH-treated mice (Figure 3G). Since activated PECs are the predominant cell type in crescentic lesions (2), we also performed immunostaining for both Claudin-1 and CD44 (marker of activated PECs). Glomerular Claudin-1 and CD44 expression was increased in all NTS-treated mice and was significantly elevated in the Podocin-Cre Klffilf mice as compared

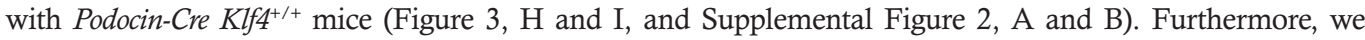
observed colocalization of CD44 with phospho-STAT3 in a subset of cells after NTS treatment in Podocin-Cre Klff $4^{\text {I/f }}$ mice (Supplemental Figure 2C). Combined, these findings suggest that the podocyte-specific loss of Klf4 contributes to increased activation of STAT3 signaling and PEC accumulation in a murine model of RPGN.

Podocyte-specific Klf4-knockdown mice on the FVB/N background develop FSGS with renal failure and reduced survival. The FVB/N mouse strain was previously reported to exhibit increased susceptibility to glomerular disease as compared with the C57BL/6J mouse strain (28), which might be partially due to enhanced constitutive activation of STAT3 signaling (29). To assess whether the podocyte-specific loss of Klf4 contributed to glomerular 
A
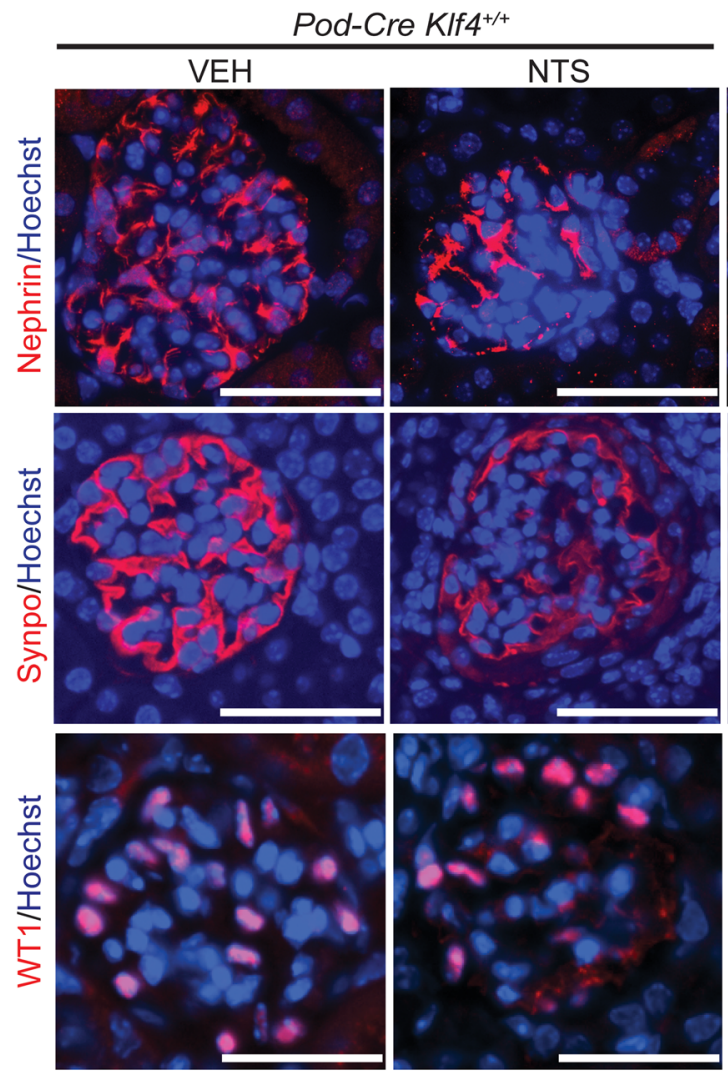

B

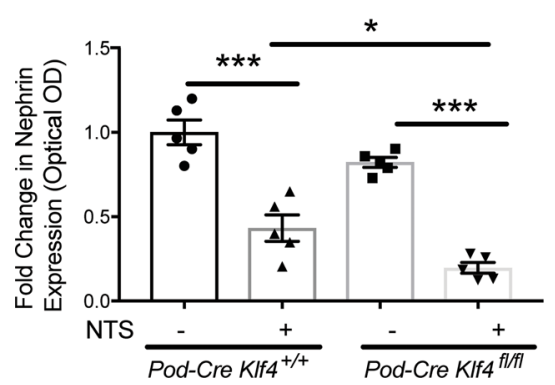

C

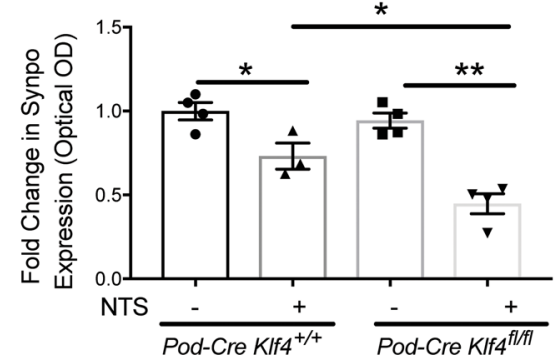

Pod-Cre KIf4t/fil
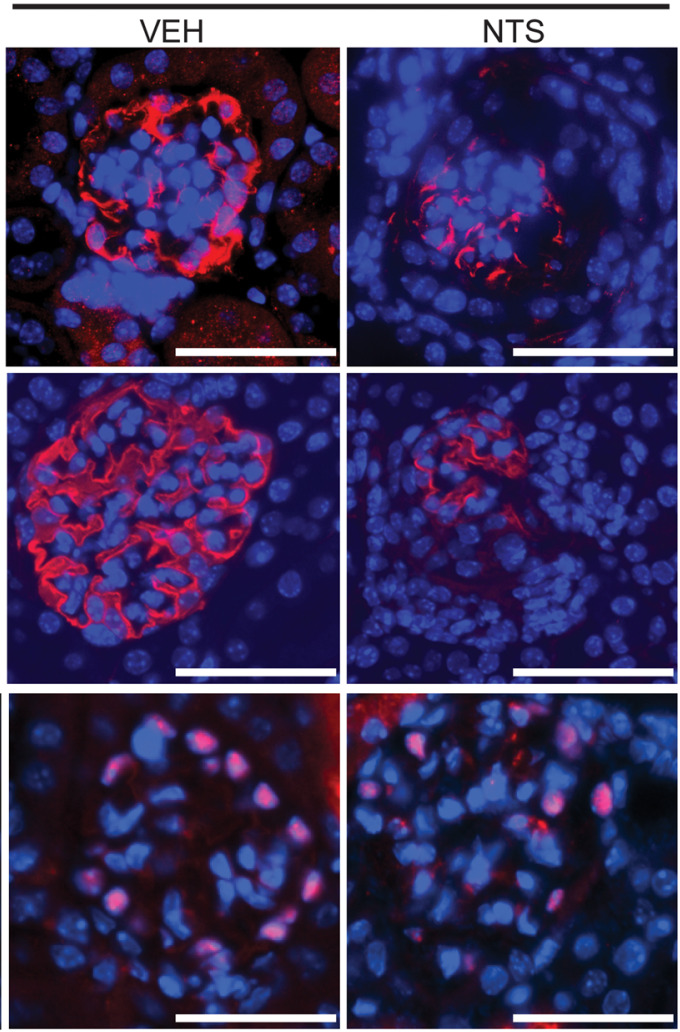

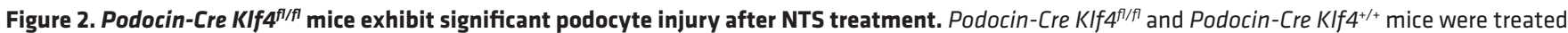
with nephrotoxic serum (NTS) or VEH for 7 days. (A) Representative images of immunostaining for Nephrin (upper panel), Synaptopodin (Synpo; middle panel) and WT1 (lower panel) ( $\times 20$ ). The glomerular region was selected and intensity of staining (OD) is shown as a relative fold change to untreated Podocin-Cre KIf4 ${ }^{+/}$mice for (B) Nephrin ( $n=5$ in each group) and (C) Synpo ( $n=3-4$ in each group). (D) Number of WT1 ${ }^{+}$cells per glomerular cross-sectional area ( $n=5$ in each group). ${ }^{*} P<0.05,{ }^{* *} P<0.01,{ }^{* *} P<0.001$; Kruskal-Wallis test with Dunn's post hoc test. Scale bars: $40 \mu \mathrm{m}$.

disease in this susceptible strain, we backcrossed Podocin-Cre Klf4/月/f mice on the C57BL/6J background to the $\mathrm{FVB} / \mathrm{N}$ background (9 generations). Podocin-Cre Klf4//fl $(\mathrm{FVB} / \mathrm{N})$ were born at the expected Mendelian frequency but, by 12 weeks of age, developed FSGS, with pseudo-crescent formation and widespread proteinaceous casts as compared with Podocin-Cre Klf4 ${ }^{+/}$littermates (Figure 4A and Table 2). Periodic acid-Schiff (PAS) staining revealed podocyte detachment with shedding into the urinary space and GEC hyperplasia, as well as mitotic GECs (Figure 4A). Ultrastructural changes by electron microscopy (EM) showed that Podocin-Cre Klf4t/lt mice exhibited extensive foot process effacement with areas of denuded basement membrane (Figure 4B). Furthermore, we noted several instances of PEC cytoplasmic extensions bridging from Bowman's capsule to a denuded glomerular tuft in the Podocin-Cre Klff//f mice by both PAS staining and EM (Figures 4, A and B). EM also revealed intracytoplasmic protein resorption droplets and enlarged, swollen mitochondria with disrupted cristae (Figure 4B). In addition, Podocin-Cre Klffl/l/ mice also experienced a significant increase in albuminuria and serum creatinine as compared with Podocin-Cre Klf4 ${ }^{+/+}$mice at 12 weeks of age (Figure 4, C and D). Evaluation 
A

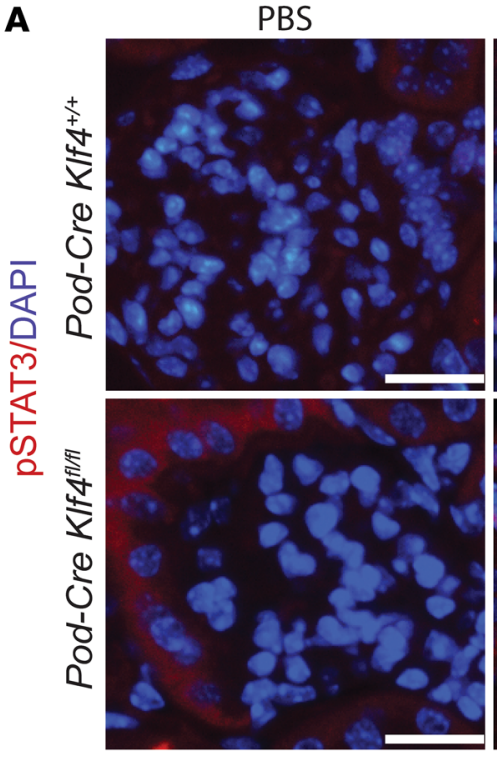

c

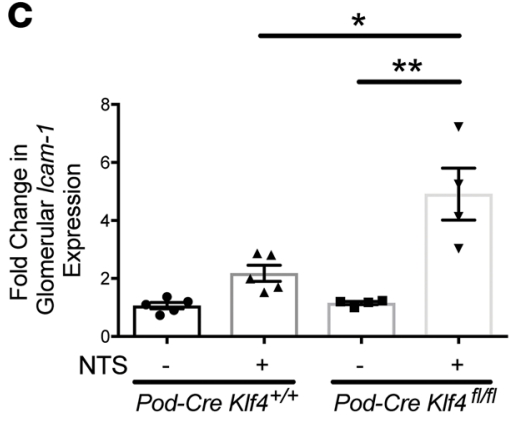

$\mathbf{F}$

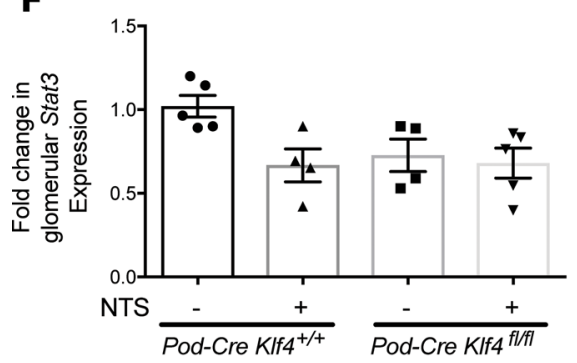

NTS

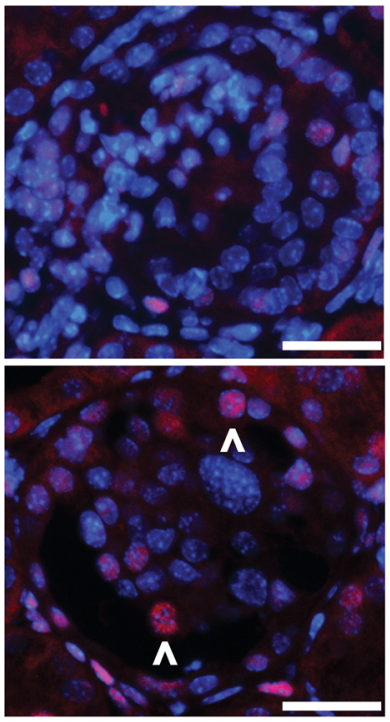

D

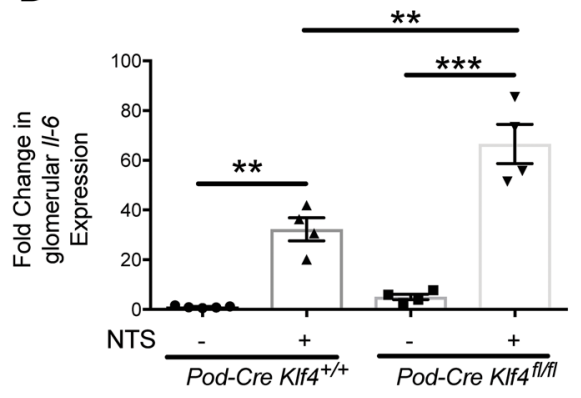

G

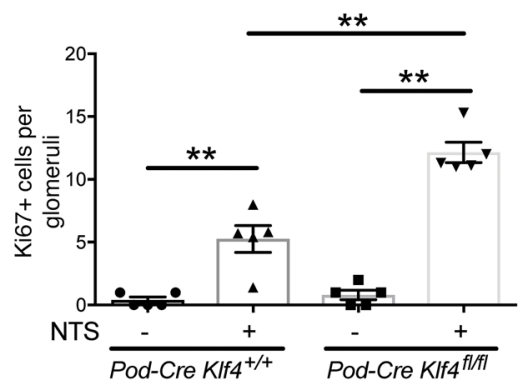

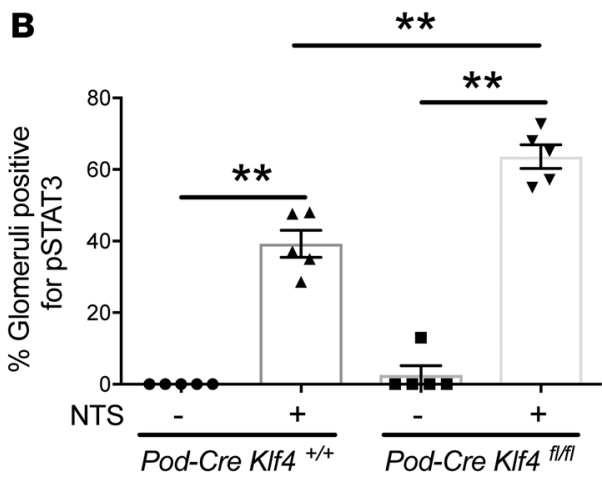

$\mathbf{E}$

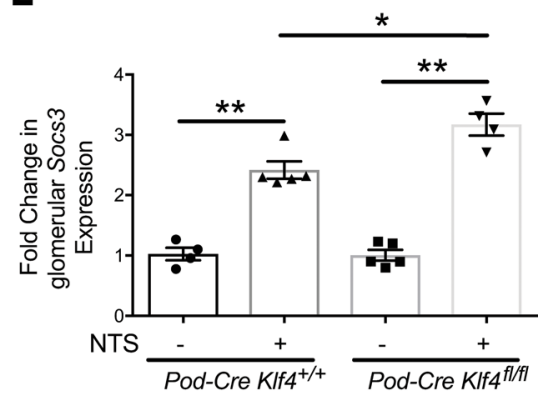

H

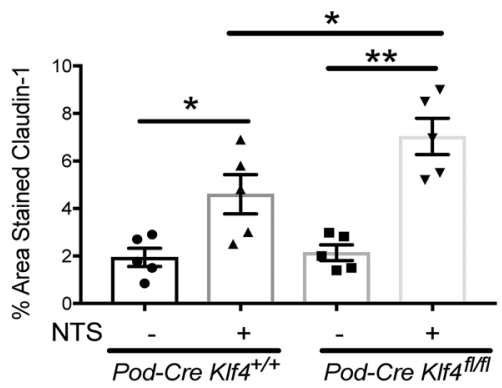

I

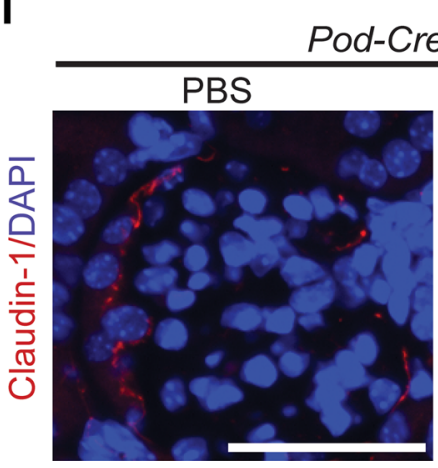

Pod-Cre KIf $4^{f|f|}$
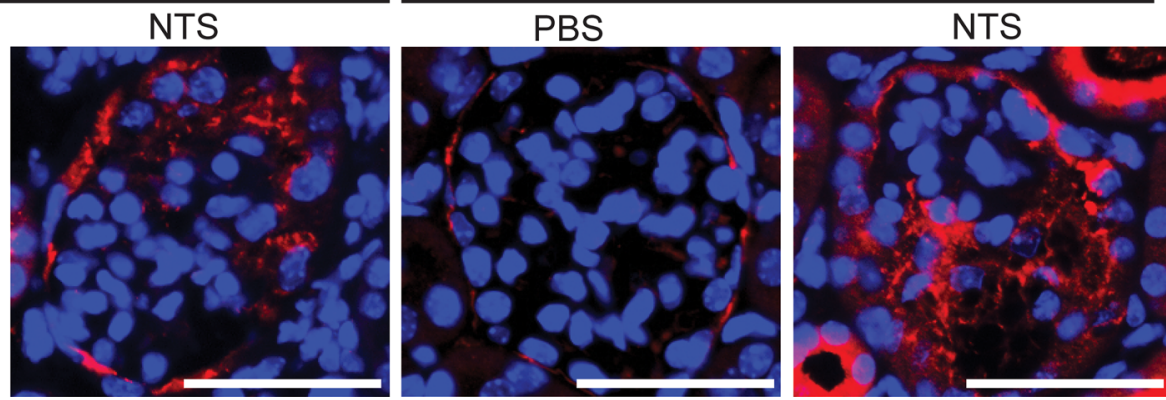
Figure 3. Podocin-Cre KIff $4^{f / f l}$ mice exhibit increased STAT3 activation and parietal epithelial cell accumulation after NTS treatment. Podocin-Cre $\mathrm{KIf}^{f / / f l}$ and Podocin-Cre KIf4 ${ }^{+/+}$mice were treated with nephrotoxic serum (NTS) or VEH for 7 days. (A) Representative images of phospho-STAT3 immunostaining. Arrowheads show nuclear phospho-STAT3 staining $(\times 20)$. Scale bars: $20 \mu \mathrm{m}$. (B) Percent of glomeruli with nuclear phospho-STAT3 staining ( $n=5$ in each group, 30 glomeruli per mouse; ${ }^{*} P<0.01$; Kruskal-Wallis test with Dunn's post hoc test). (C) Icam-1, (D) II-6, (E) Socs3, and (F) Stat3 mRNA expression in glomeruli $\left(n=4-5\right.$ in each group; ${ }^{*} P<0.05$, ${ }^{* *} P<0.01$, ${ }^{* * *} P<0.001$; Kruskal-Wallis test with Dunn's post hoc test). (G) Immunostaining for Ki67 was performed, and the number of Ki67+ cells per glomeruli were determined $\left(n=5\right.$ in each group, 30 glomeruli per mouse; ${ }^{* *} P<0.01$; Kruskal-Wallis test with Dunn's post hoc test). ( $\mathbf{H}$ and $\mathbf{I})$ Representative images of Claudin-1 immunostaining $(\times 20)$ with quantification of percent area stained ( $n=5$ in each group, 30 glomeruli per mouse; ${ }^{*} P<0.05,{ }^{*} P<0.01$; Kruskal-Wallis test with Dunn's post hoc test). Scale bars: $40 \mu \mathrm{m}$.

of younger Podocin-Cre Klf4l/fl mice revealed normal urinary albumin excretion up until approximately 4 weeks of age, and the development of mild proteinuria starting at 5-6 weeks of age (Supplemental Figure 3A, inset), with marked albuminuria by 8-9 weeks of age (Supplemental Figure 3A). Furthermore, there was not a rise in serum creatinine at 5 weeks of age (Supplemental Figure 3B). These findings suggest that podocyte injury was likely not secondary to a loss of Klf4 during podocyte development — rather, they suggest that Klf4 is required for maturation and maintenance of mature podocyte differentiation markers. Finally, we observed a significant increase in mortality in the Podocin-Cre Klffl/fl mice as compared with Podocin-Cre Klf4 ${ }^{+/+}$, with less than a third surviving at 52 weeks of age (Figure 4E).

In addition to the renal dysfunction and FSGS observed in the Podocin-Cre Klf4lffl mice on the FVB/N background, Nephrin, Synaptopodin, and WT1 mRNA and protein expression were also significantly reduced as compared with Podocin-Cre $\mathrm{Klf4}^{+/+}$littermates at 12 weeks of age (Figure 5, A-C). However, these changes in Nephrin and Synaptopodin mRNA were not statistically significant at 5 weeks of age (Supplemental Figure 3C), suggesting that podocyte injury is more likely a result of impaired maintenance of mature podocyte differentiation markers in adult Podocin-Cre Klf4//fl mice. Finally, immunostaining for p57 - which has previously been shown to be highly expressed in mature, differentiated podocytes and reduced in cellular FSGS lesions (30) - demonstrated a marked decrease in Podocin-Cre Klf4 ${ }^{f / f l}$ mice as compared with Podocin-Cre Klf4 ${ }^{+/+}$littermates at 12 weeks of age (Figure 5D). These data suggest that the knockdown of Klf4 in podocytes on the FVB/N background contributed to the loss of differentiation markers and podocyte injury, leading to progressive and significant albuminuria and FSGS with GEC proliferation.

Podocyte-specific Klf4 knockdown mice on the FVB/N background exhibit increased STAT3 activation and GEC proliferation. STAT3 activation has been implicated in the development of various subtypes of FSGS $(31,32)$. To assess whether activation of STAT3 signaling was associated with the FSGS lesions observed in Podocin-Cre $K l f 4^{l / f l}(\mathrm{FVB} / \mathrm{N})$ mice, we performed immunostaining for phospho-STAT3. We observed a small percentage of glomeruli with phospho-STAT3 ${ }^{+}$cells in Podocin-Cre Klf4 $4^{+/+}$mice, with a marked increase in Podocin-Cre Klffl/fl mice (Figure 6A). In these Podocin-Cre Klf4l/fl mice, glomerular phospho-STAT3 was expressed in both podocytes and PECs, as evidenced by its coexpression with the podocyte marker Nestin and the PEC markers Claudin-1 and CD44 (Figure 6, B and C, and Supplemental Figure 4). Since Podocin-Cre Klffl/fl mice exhibited a significant increase in epithelial cells in the Bowman's space (i.e., pseudo-crescent formation), we performed immunofluorescence for Ki67 to determine if these cells were proliferating. We observed a marked increase in Ki67 staining in the periphery of glomeruli of Podocin-Cre Klf4 $4^{l / f l}$ mice as compared with Podocin-Cre Klf4 $4^{+/+}$mice (Figure 6D). To further delineate the source of proliferating cells, we costained Ki67 with the PEC marker Claudin-1. We observed a significant increase in glomerular Claudin-1 expression in Podocin-Cre Klf4l/fl mice as compared

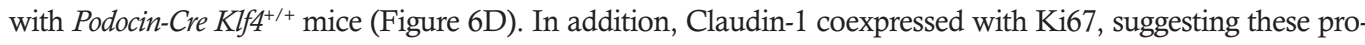
liferating GECs were likely of PEC origin (Figure 6D). We validated these findings by staining for CD44 and showing that Podocin-Cre $\mathrm{Klf4}^{+/+}$mice exhibit no glomerular CD44 expression, which was markedly increased in Podocin-Cre Klf4 $4^{\text {Ilfl }}$ mice (Supplemental Figure 4). Downstream targets of STAT3 activation (II-6, Socs3, Icam-1) were also significantly increased in the glomeruli of Podocin-Cre Klf4t/fl mice as compared with Podocin-Cre Klf4 ${ }^{+/+}$ mice (Figure 6E). No significant changes were observed in Stat3 mRNA expression (Figure 6E). Finally, we used the selective STAT3 inhibitor S3I-201, which binds to the SH2 domain $(33,34)$, to determine if inhibiting STAT3 signaling partially rescues the phenotype of the Pod-Cre Klffl/ft $(\mathrm{FVB} / \mathrm{N})$ mice. We randomized PodocinCre Klffl/fl mice at 7 weeks of age that had dipstick-positive proteinuria to receive either S3I-201 (i.p., $10 \mathrm{mg} /$ $\mathrm{kg}, 3$ times weekly) or DMSO for a total of 3 weeks and measured albuminuria weekly. We observed that S3I201-treated Podocin-Cre Klffl/fl mice exhibited significantly less albuminuria at 11 weeks of age as compared with the DMSO-treated Podocin-Cre Klffl/fl mice (Figure 6F). Collectively, these data suggest that the FSGS lesions observed in the Podocin-Cre Klf4/l/f mice on the FVB/N background might be due to enhanced activation of both podocyte and PEC/STAT3 signaling, leading to dysregulated PEC proliferation and FSGS. 
A
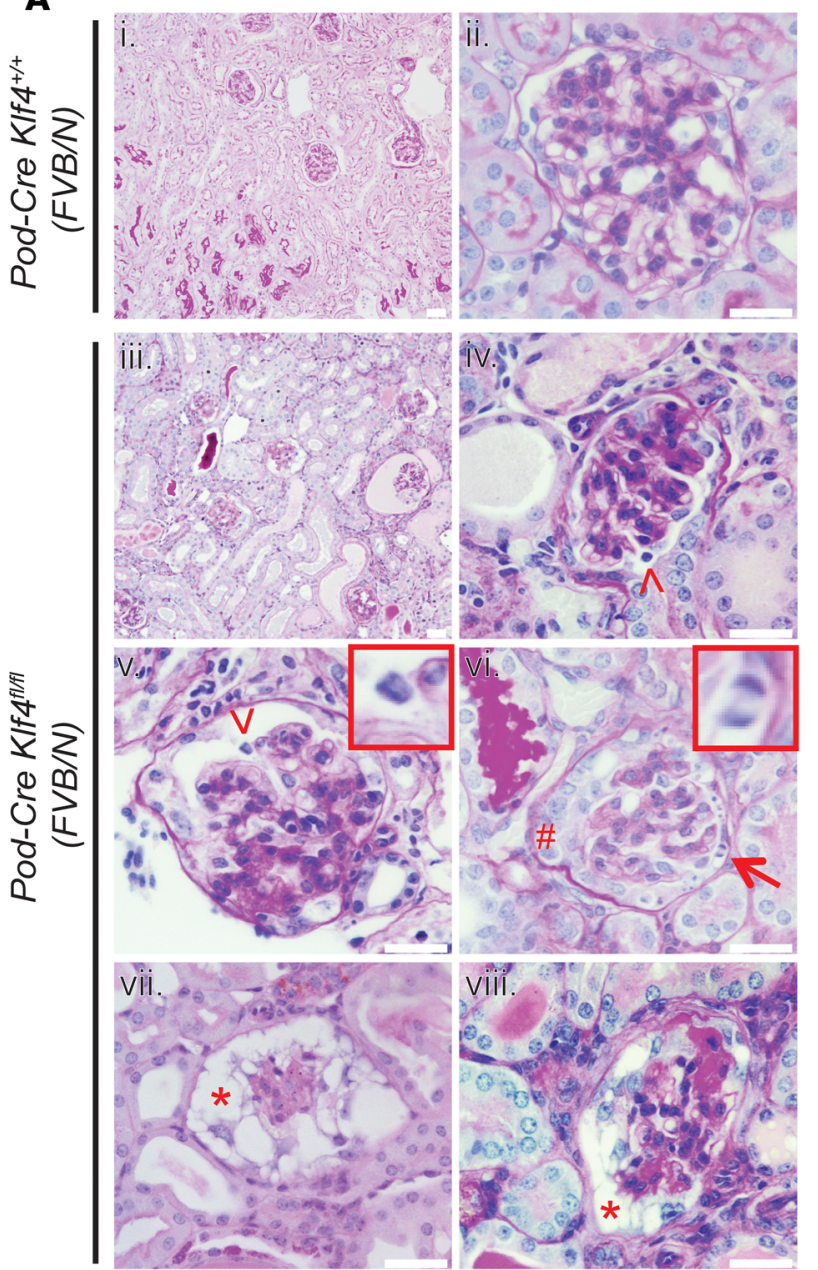

C

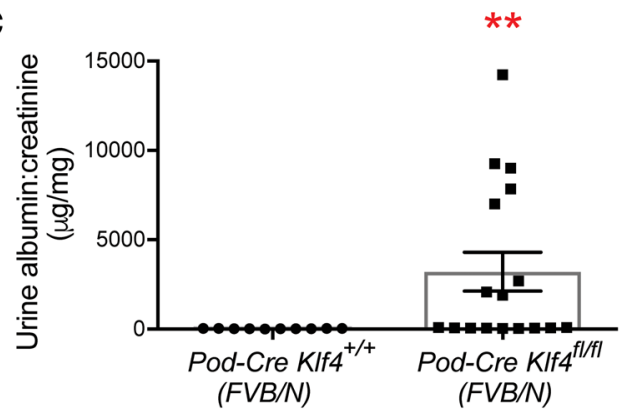

D

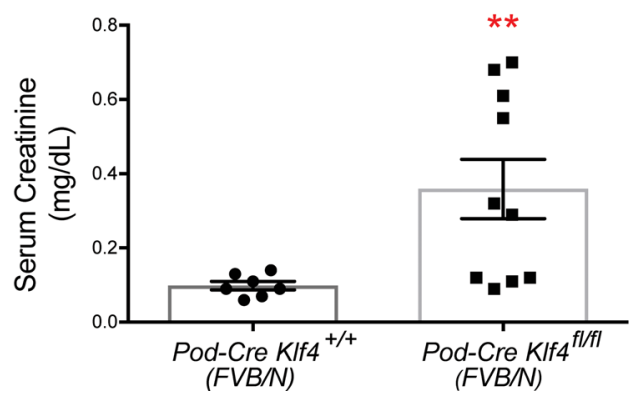

B
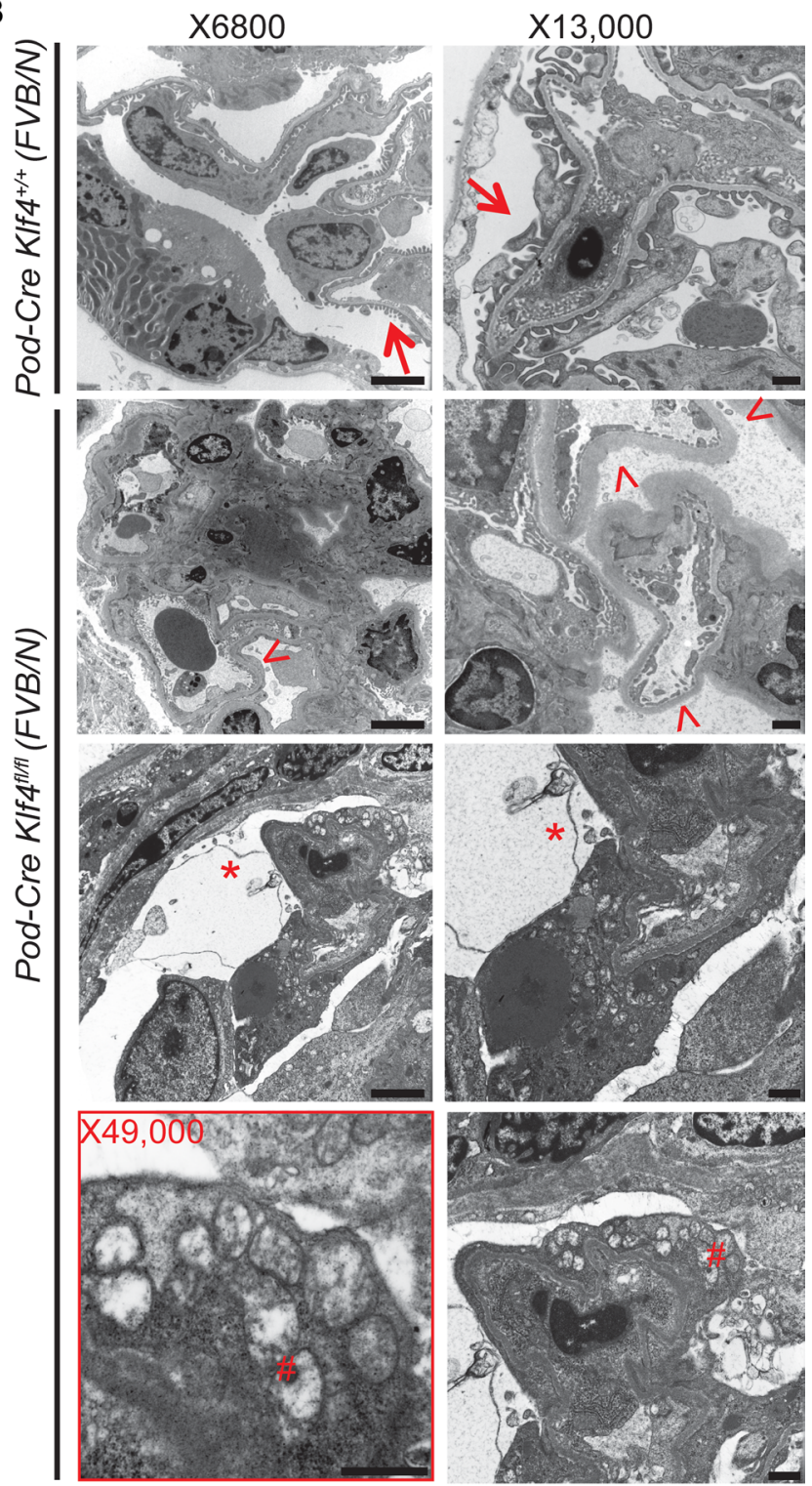

E

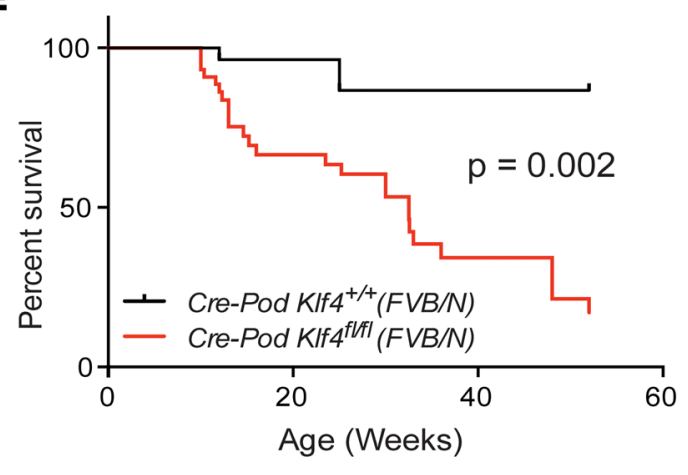

Figure 4. Podocin-Cre KIf4 ${ }^{f / f l}$ (FVB/N) mice exhibit FSGS, renal failure and reduced survival. (A) Representative images of periodic acid-Schiff (PAS) staining for Podocin-Cre KIf4 ${ }^{+/+}(\mathrm{FVB} / \mathrm{N})$ mice (i: $\times 10$ and ii: $\left.\times 40\right)$ and Podocin-Cre KIf4//fl (FVB/N) mice (iii: $\times 10$ and iv-viii: $\times 40$ ). Representative images show (iv-v) podocyte detachment (arrowhead, inset 120x), (vi) extracapillary proliferation (pound sign), (vi) glomerular epithelial cell mitosis (arrow, inset 120x), and (vii-viii) parietal epithelial cytoplasmic extensions (asterisk). Scale bars: $50 \mu \mathrm{m}$ (10x images), $20 \mu \mathrm{m}$ (40x images). (B) Electron microscopy was performed to assess ultrastructural changes in podocyte morphology $(\times 6,800 ; \times 13,000$; and $\times 49,000$; scale bars: $3 \mu \mathrm{m}, 750 \mathrm{~nm}$, and $600 \mathrm{~nm}$, respectively). 
Representative images show normal upright foot processes (arrow), podocyte effacement and denuded basement membrane (arrowhead), parietal epithelial cytoplasmic extensions (asterisk), and enlarged mitochondria with disrupted cristae (pound sign). (C) Albuminuria (urine albumin/creatinine) ( $n=10$ in Podocin-Cre KIf $4^{+/+}$and 17 in Podocin-Cre KIf $4^{f / / f l} ;{ }^{* *} P<0.01$; Mann-Whitney $U$ test) and (D) serum creatinine at 12 weeks of age $\left(n=7\right.$ in Podocin-Cre KIf4 ${ }^{+/+}$

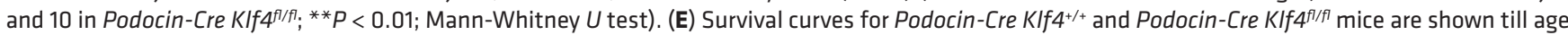
52 weeks ( $n=30-40$ in each group; $P=0.002$; log-rank [Mantel-Cox] test).

FVB/N mice exhibit basal levels of constitutively activated STAT3 compared with C57BL/6J mice. Since previous reports demonstrated that FVB/N mice exhibit some activation of STAT3 signaling at baseline (28), we postulated whether enhanced activation of STAT3 signaling might be contributing to proliferative FSGS lesions observed in the Podocin-Cre Klffl/fl mice on the FVB/N background as compared the C57BL/6J background. We observed an increase in phospho-STAT3 ${ }^{+}$cells in the glomeruli of WT FVB/N mice as compared with WT C57BL/6J mice with colocalization to a podocyte-specific marker, WT1 (Supplemental Figure 5, A and B). In comparison, we observed no phospho-STAT3 ${ }^{+}$cells in the glomeruli of the WT C57BL/6J mice (Supplemental Figure 5, A and B). In addition, no significant differences were observed in glomerular Stat 3 mRNA expression between WT mice of both strains (Supplemental Figure 5C). Similarly, Podocin-Cre Klf4t/fl (FVB/N) mice exhibited an increase in phospho-STAT3 expression as compared with Podocin-Cre Klffl/fl (C57BL/6J) mice by Western blot (Supplemental Figure 5, D and E). Taken together, these data suggest that increased activation of STAT3 signaling might contribute to the development of FSGS in the Podocin-Cre Klffl/ft mice on the FVB/N background, as compared with the C57BL/6J background.

KLF4 knockdown in podocytes results in cell cycle reentry, mitotic catastrophe, reduced podocyte survival, and increased paracrine IL-6 signaling. To assess the mechanism by which the loss of KLF4 exacerbated podocyte injury in vivo, we generated human podocytes with both partial (KLF4-shRNA21) and near complete (KLF4-shRNA22) knockdown for KLF4 (Supplemental Figures 6, A and B), in addition to a control cell line (Scr-shRNA22). Prior studies have demonstrated that KLF4 is highly expressed in differentiated human podocytes, as demonstrated by increased expression of $\mathrm{KLF} 4$ in differentiated, nonpermissive $\left(37^{\circ} \mathrm{C}\right)$, as compared with undifferentiated, permissive $\left(33^{\circ} \mathrm{C}\right)$ conditions (19). As such, at permissive conditions $\left(33^{\circ} \mathrm{C}\right)$, none of the 3 podocyte lines exhibited significant changes in survival. However, under nonpermissive conditions $\left(37^{\circ} \mathrm{C}\right)$, both $K L F 4$-shRNA22 and KLF4-shRNA21 podocytes demonstrated reduced survival as compared with $S c r$-shRNA cells at day 3, 7, 10, and 14 days of differentiation (Figure 7A). Interestingly, $K L F 4$-shRNA22 podocytes, which had more efficient knockdown of KLF4, exhibited more cell death, thereby suggesting that the efficiency of KLF4 knockdown contributes to cell survival (Figure 7A). Furthermore, we measured cell viability using the 3-(4, 5-dimethylthiazolyl-2)-2, 5-diphenyltetrazolium bromide (MTS) assay, which confirmed a significant decrease in podocyte viability in KLF4-shRNA22 as compared with Scr-shRNA under nonpermissive conditions (Figure 7B). To investigate the basis of this podocyte loss, we performed cell cycle analysis at day 3 of differentiation and noted a marked decrease in percentage of cells in $\mathrm{G}_{0} / \mathrm{G}_{1}$ phase with a concurrent increase in both $\mathrm{S}$ and $\mathrm{G}_{2} / \mathrm{M}$ phases in KLF4-shRNA22 as compared with $S c r$-shRNA podocytes (Figure 7C). This cell-cycle reentry was confirmed by demonstrating a significant increase in both CCNE1 and CCNA2, as well as a decrease in CCND1 expression in KLF4-shRNA22 as compared with Scr-shRNA podocytes (Figure 7D). Similarly, we observed an increase in Ki67 staining (Supplemental Figure 6C), as well as an increase in binucleated podocytes (Supplemental Figure 6D) by day 3 of differentiation in KLF4-shRNA22 as compared with $S c r$-shRNA podocytes. We postulated that this dysregulated cell cycle progression might be due to the loss of cell cycle inhibition, as KLF4 has been shown to transcriptionally upregulate $p 57$ (35). Interestingly, we observed nearly a 10 -fold reduction in $p 57$ expression in KLF4-shRNA22 as compared with Scr-shRNA podocytes (Figure 7D).

To further demonstrate that the loss of podocyte differentiation markers contributed to reduced cell survival in KLF4-shRNA22 podocytes, we performed immunostaining for F-actin and observed a loss of actin stress fiber formation in KLF4-shRNA22 podocytes as compared with Scr-shRNA podocytes at 3 and 5 days of differentiation (Supplemental Figure 6D and Figure 7E, respectively). Furthermore, nuclei of KLF4-shRNA22 podocytes were noted to be small and contracted with multiple apoptotic bodies (Figure 7E). We confirmed this increased apoptosis in KLF4-shRNA22 podocytes as compared with Scr-shRNA podocytes using FACS analysis with annexin $\mathrm{V}$ and propidium iodide staining (Figure $7 \mathrm{~F}$ ).

Finally, to assess the role of STAT3 signaling in differentiated podocytes, we measured phospho-STAT3 expression under permissive and nonpermissive conditions. In Scr-shRNA podocytes, phospho-STAT3 expression was significantly increased at $33^{\circ} \mathrm{C}$ as compared with $37^{\circ} \mathrm{C}$, suggesting suppression 
Table 2. Quantification of histologic changes in FVB/N model

\begin{tabular}{|c|c|c|c|c|c|}
\hline Podocin-Cre KIf4+/+ & 0.0 & 0.0 & 0.0 & 0.0 & 0.0 \\
\hline
\end{tabular}

${ }^{A} P<0.05$. Tubulointerstitial scoring: 0 , none; $1,<10 \% ; 2,10 \%-25 \% ; 3,>25 \%-50 \%$.

of STAT3 signaling in the differentiated state (Figure 8A). Interestingly, KLF4-shRNA22 podocytes exhibited reactivation in STAT3 signaling compared with $S c r$-shRNA podocytes under nonpermissive conditions, as demonstrated by increased IL- 6 and phospho-STAT3 expression (Figure 8, B and C). To determine whether pharmacological inhibition of STAT3 signaling might improve cell survival, we treated KLF4-shR $N A 22$ podocytes with the STAT3 inhibitor S3I-201 under nonpermissive conditions. We observed a partial restoration in survival of S3I-201-treated KLF4-shRNA22 podocytes as compared with DMSO-treated KLF4-shRNA22 podocytes at 72 hours after treatment (Figure 8D).

We previously demonstrated that the podocyte-specific loss of KLF4 contributed to the activation of STAT3-induced PEC proliferation (Figure 6). To determine the mechanism by which KLF4 knockdown in podocytes activates STAT3 signaling in PECs, we measured IL-6 levels in the supernatant of Scr-shRNA and KLF4-shRNA22 podocytes and observed a significant increase in KLF4-shRNA22 podocytes as compared with $S c r$-shRNA podocytes (Figure $8 \mathrm{E}$ ). Since previous studies have demonstrated that IL-6 is an activator of STAT3 signaling (36), we treated primary cultured PECs with the supernatant from KLF4-shRNA22 and Scr-shRNA podocytes for 24 hours. Interestingly, phospho-STAT3 expression was increased in PECs treated with supernatant from KLF4-shRNA22 podocytes as compared with Scr-shRNA podocytes (Figure 8F). We subsequently treated cultured PECs with supernatant from Scr-shRNA and KLF4-shRNA22 podocytes, and in combination with either the STAT3 inhibitor S3I-201 or DMSO. After 24 hours of treatment, we observed a significant increase in proliferation in PECs treated with supernatant from KLF4-shRNA22 podocytes as compared with $S c r$-shRNA podocytes (Figure 8G). Interestingly, concurrent treatment with S3I-201 attenuated proliferation in PECs exposed to supernatant from KLF4-shRNA22 podocytes (Figure 8G).

Taken together, our findings suggest that the knockdown of KLF4 in differentiated podocytes activates STAT3 signaling, leading to a loss of mature podocyte differentiation markers and eventual mitotic catastrophe and podocyte death, while triggering the release of IL-6 to induce STAT3 activation in PECs.

KLF4 overexpression reduces LIF-induced STAT3 signaling and cell cycle progression. To assess whether the overexpression of $K L F 4$ has a protective role in podocytes, we generated human podocytes with stable overexpression of KLF4 (LentiORF-KLF4) and control (LentiORF-RFP) (Figure 9A). To evaluate cell cycle progression, we performed FACS analysis under permissive conditions and determined an increase in the percentage of cells in $\mathrm{G}_{0} / \mathrm{G}_{1}$ phase in LentiORF-KLF4 podocytes as compared with LentiORF-RFP podocytes (Figure 9B). Next, to evaluate the effect of KLF4 overexpression on STAT3 activation, we treated differentiated LentiORF-KLF4 and LentiORF-RFP podocytes with leukemia inhibitor factor (LIF), a known STAT3 activator (37), for 15 minutes and subsequently measured $I L-6$ expression. $I L-6$ expression was significantly increased after LIF treatment in LentiORF-RFP podocytes as compared with LentiORF-KLF4 podocytes, suggesting that KLF4 induction inhibited the early effects of STAT3 signaling (Figure 9C). As overexpression of KLF4 in neurons has been demonstrated to similarly suppress downstream signaling by binding to phospho-STAT3 (21), we sought to validate this interaction in podocytes. We immunoprecipitated KLF4 with anti-KLF4 antibody in LentiORF-RFP and LentiORF-KLF4 podocytes and subsequently immunoblotted for phospho-STAT3 to demonstrate that there is enhanced binding of KLF4 to phospho-STAT3 upon KLF4 overexpression (Figure 9D).

To demonstrate reversibility, restoration of KLF4 expression in KLF4-shRNA22 podocytes markedly improved cell survival under nonpermissive conditions (Figure 9E). In addition, treatment of cultured PECs with supernatant from LentiORF-KLF4 podocytes did not alter cell proliferation as compared with supernatant from LentiORF-RFP podocytes, contrary to the increase in PEC proliferation with exposure to supernatant from KLF4-shRNA22 podocytes (Supplemental Figure 6E and Figure 8G). Collectively, these data suggest that KLF4 is critical to the maintenance of mature differentiation markers and cell survival by preventing the activation of STAT3 signaling. 


\section{A}
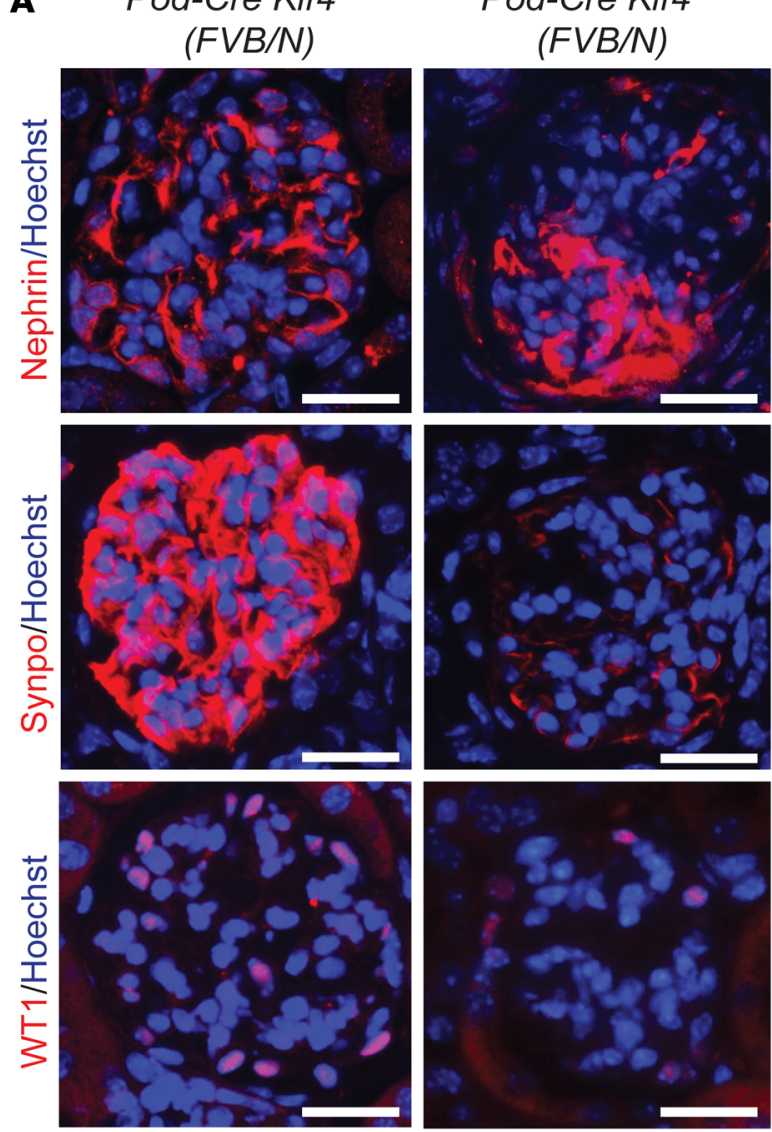

C

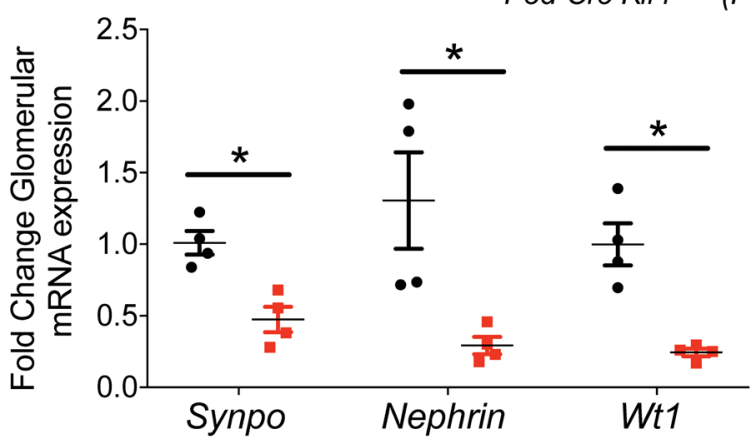

Figure 5. Podocin-Cre KIff ${ }^{f / f l}$ (FVB/N) mice exhibit significant podocyte injury. (A) Representative images of immunostaining for Nephrin (upper panel), Synaptopodin (Synpo) (middle panel), and WT1 (lower panel) in Podocin-Cre KIf4 $4^{f / f l}(\mathrm{FVB} / \mathrm{N})$ and Podocin-Cre Klf4 ${ }^{+/+}(\mathrm{FVB} / \mathrm{N})$ mice $(\times 20)$. (B) The glomerular region was selected, and intensity (OD) was measured and quantified as a relative fold change to untreated Podocin-Cre KIff ${ }^{+/+}$ (FVB/N) mice for Nephrin (upper panel) and Synpo (middle panel) $(n=$ 5 in each group, 30 glomeruli per mouse). The number of $\mathrm{WT}^{+}$cells per glomerular cross-sectional area (lower panel) $(n=4-5$ in each group, 30 glomeruli per mouse). ${ }^{*} P<0.05$; ${ }^{*} P<0.01$; Mann-Whitney $U$ test. (C) Nephrin, Synaptopodin, and Wt1 mRNA expression levels in glomeruli ( $n$ $=4$ in each group; ${ }^{*} P<0.05$; Mann-Whitney $U$ test). (D) Representative images of $\mathrm{p} 57 \mathrm{immunostaining} \mathrm{(top} \mathrm{panel).} \mathrm{Quantification} \mathrm{of} \mathrm{percent}$ $p 57^{+}$cells per glomeruli (bottom panel) $\left(n=5\right.$ in each group; ${ }^{* *} P<0.01$; Mann-Whitney $U$ test). Scale bars: $40 \mu \mathrm{m}$.
B
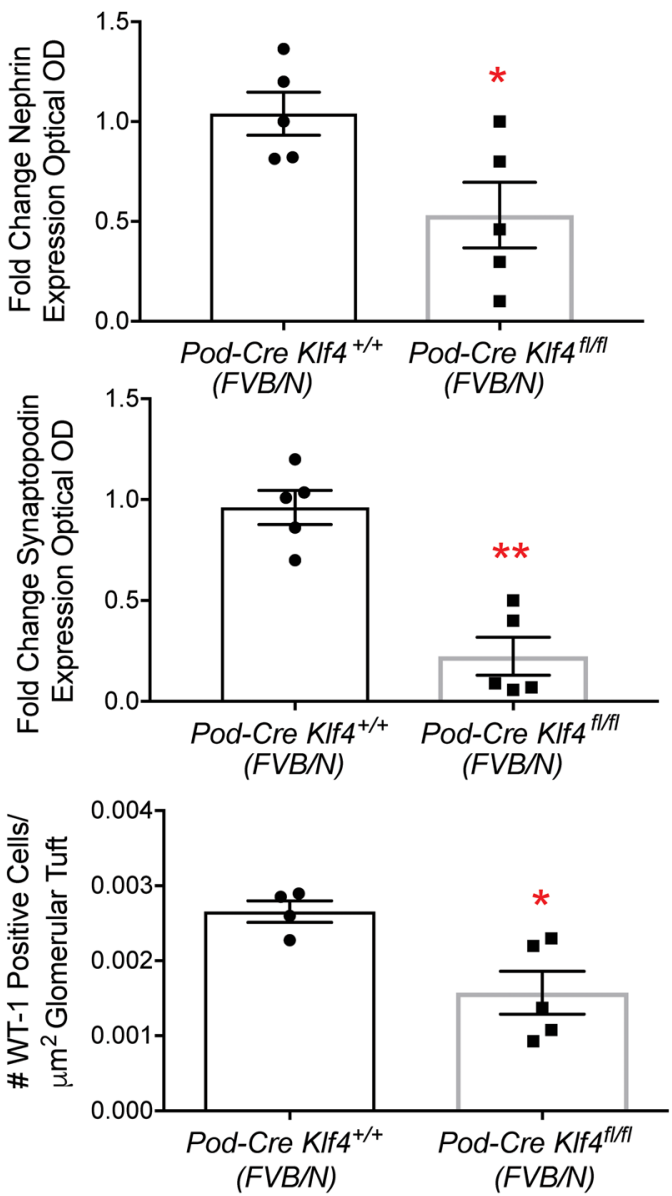

D
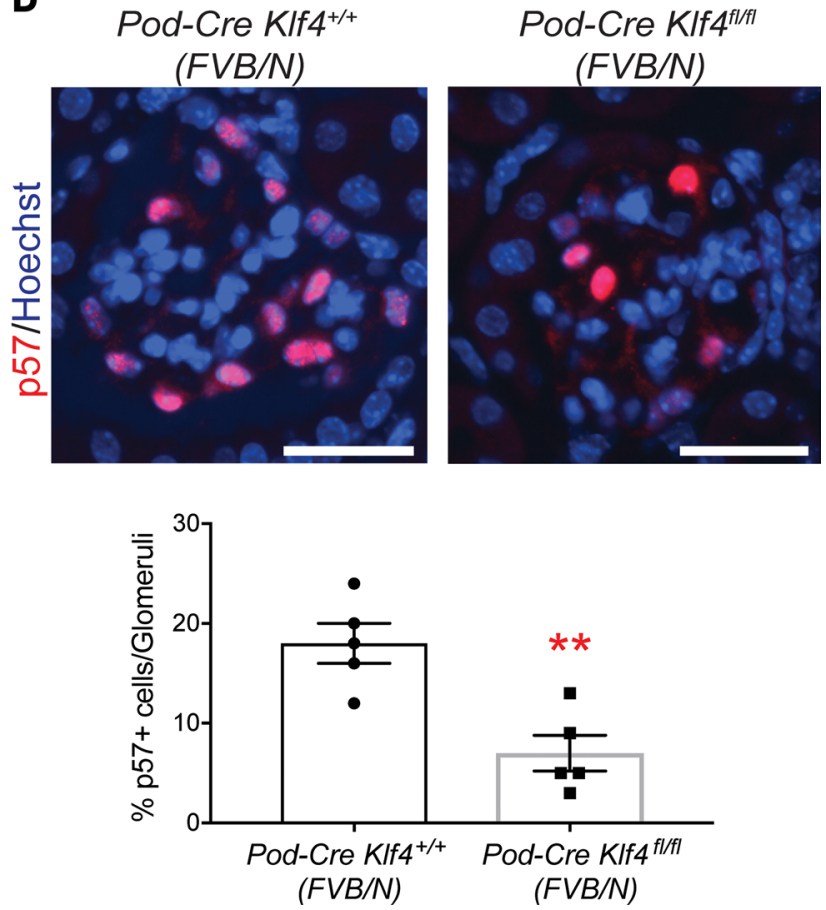
A

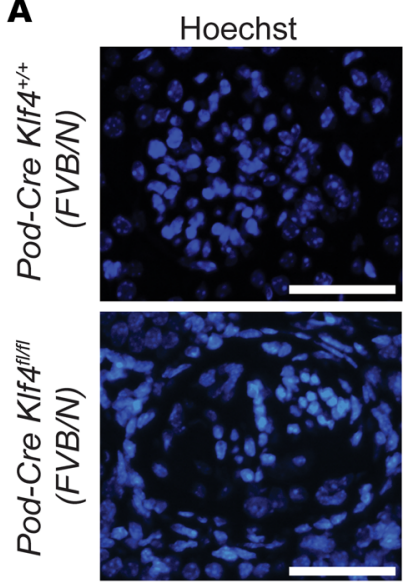

B

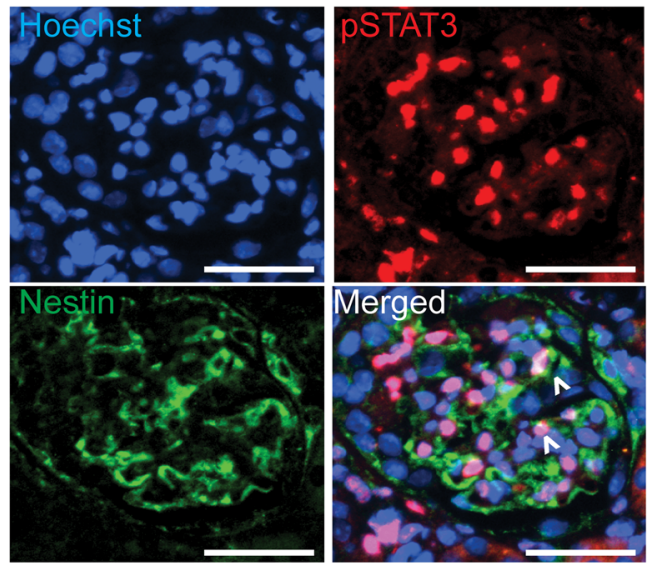

D Pod-Cre KIf4 $4^{+/+}(F V B / N)$ Pod-Cre KIffil/f $(F V B / N)$
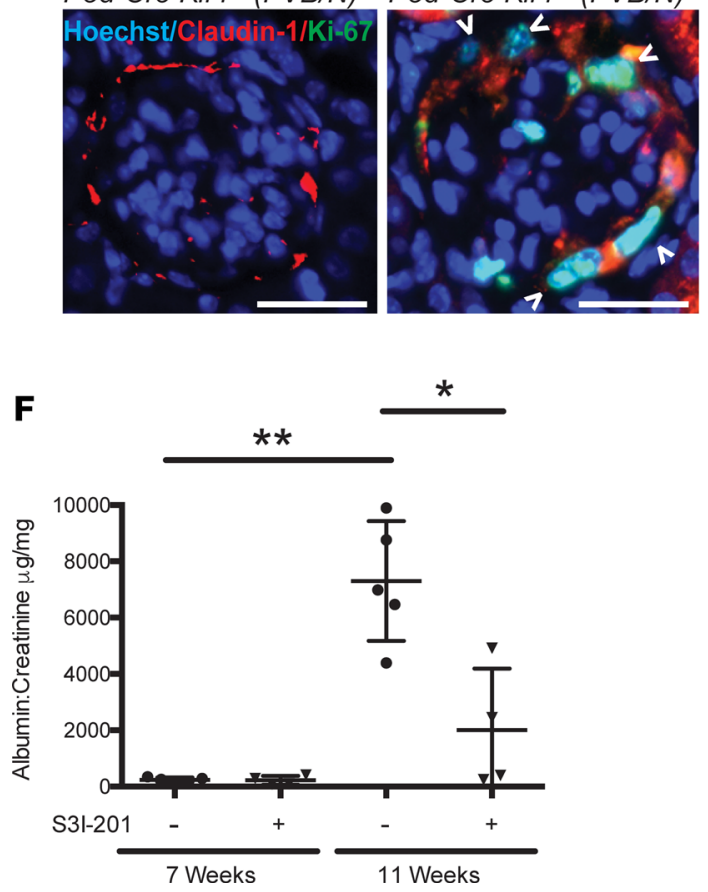

pSTAT3

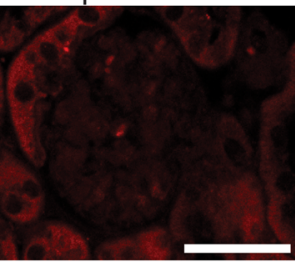

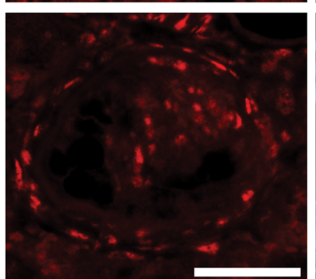

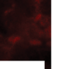

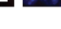 \\ .}

c

C
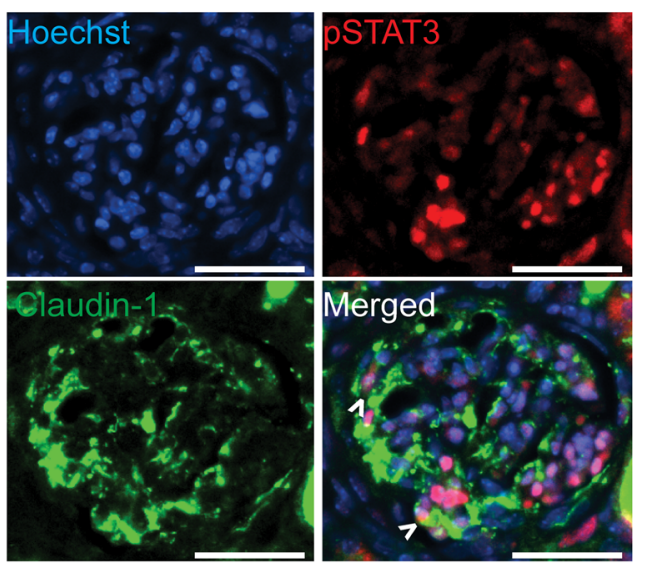

\section{E}

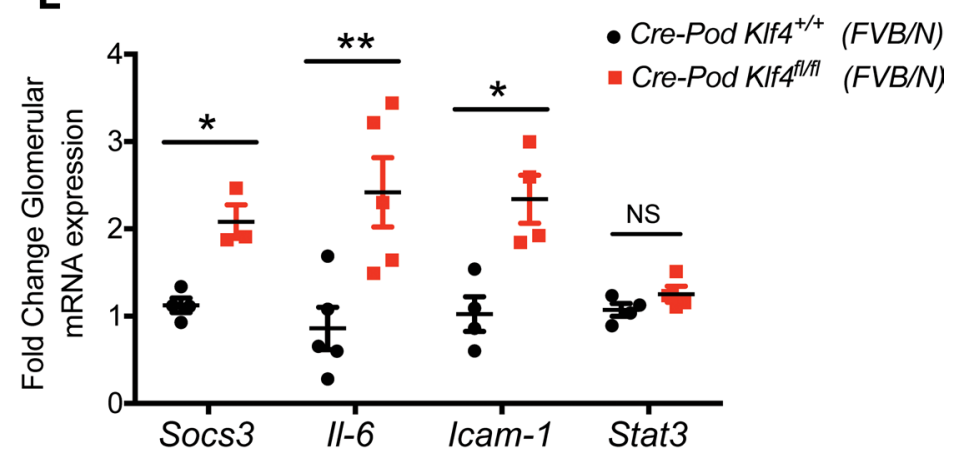

Figure 6. Podocin-Cre KIf4/f/fl (FVB/N) mice exhibit STAT3 activation and parietal epithelial cell proliferation. (A) Representative images of immunostaining for phospho-STAT3 in Podocin-Cre KIf $4^{f / / f l}(\mathrm{FVB} / \mathrm{N})$ and Podocin-Cre Klf4 ${ }^{+/+}(\mathrm{FVB} / \mathrm{N})$ mice (left panel) $(\times 20)$. Percent of glomeruli with nuclear phospho-STAT3 staining (right panel) ( $n=5-9$ in each group, 30 glomeruli per mouse; ${ }^{* *} P<0.01$; Mann-Whitney $U$ test). Representative images for phospho-STAT3 and Nestin (B) and phospho-STAT3 and Claudin-1 (C) coimmunostaining in Podocin-Cre KIf $4^{f / f l}$ mice $(\times 20)$. Arrowheads demonstrate coexpression of nuclear phospho-STAT3 with Nestin and Claudin-1. (D) Representative images for Claudin-1 and Ki67 immunostaining $(\times 20)(n=5$ per group). Arrowheads demonstrate coexpression of nuclear Ki67 with Claudin-1. (E) Socs3, II-6, Icam-1, and Stat3 mRNA expression levels in glomeruli ( $n=4-5$ in each group; ${ }^{*} P<0.05$, ${ }^{* *} P<0.01$; Mann-Whitney $U$ test). (F) Podocin-Cre KIf4l/ffl (7 weeks) with dipstick-positive proteinuria were randomized to receive $S 31-201(10 \mathrm{mg} / \mathrm{kg})$ or DMSO for 3 weeks. Albuminuria (urine albumin/creatinine) at 7 weeks (pretreatment) and at 11 weeks of age (after treatment) $\left(n=4-5\right.$ in each group, ${ }^{*} P<0.05$, ${ }^{* *} P$ $<0.01$, Kruskal-Wallis test with Dunn's post hoc test). Scale bars: $40 \mu \mathrm{m}$. 
A

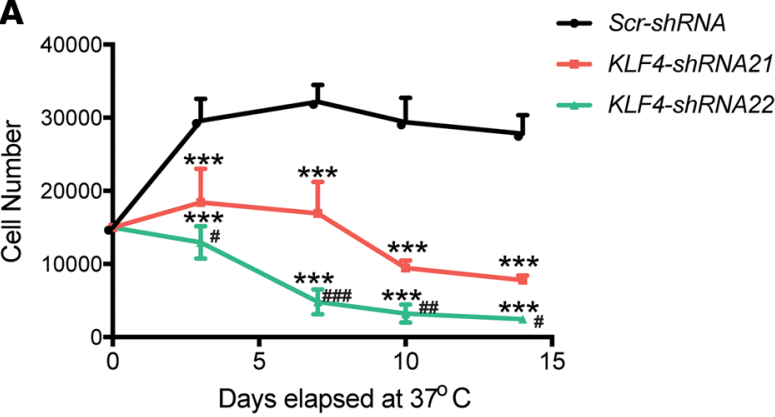

B

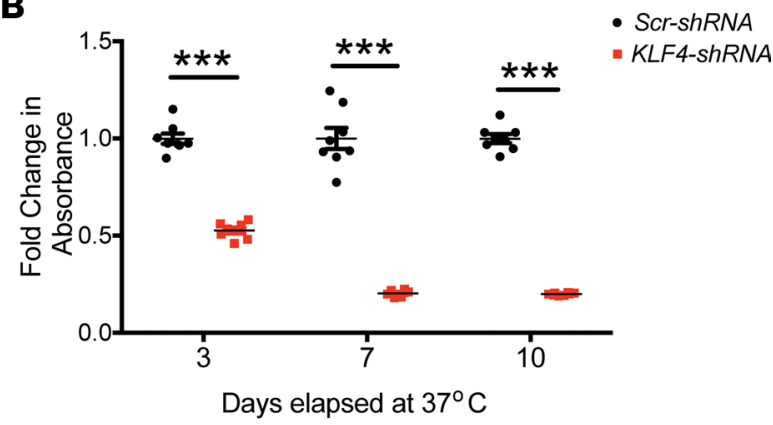

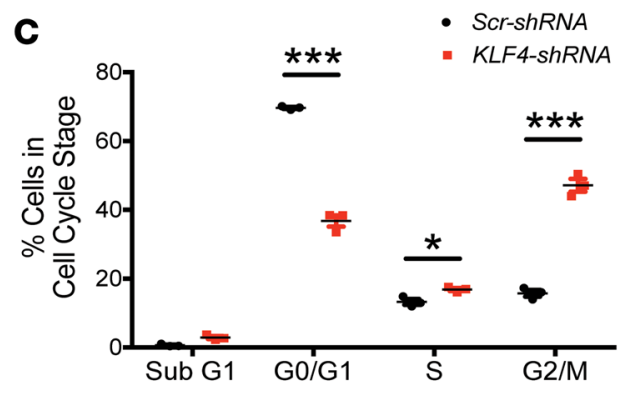

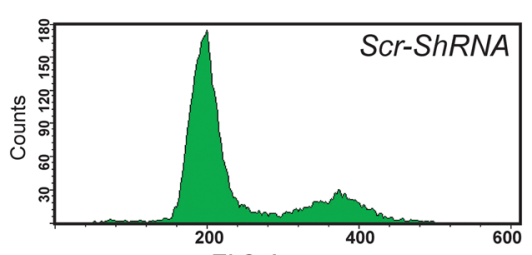

FL2-Area

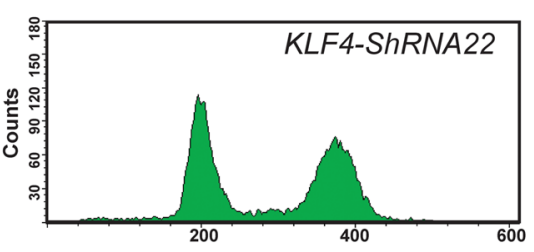

FL2-Area

\section{D \\ - Scr-shRNA \\ - KLF4-shRNA22}

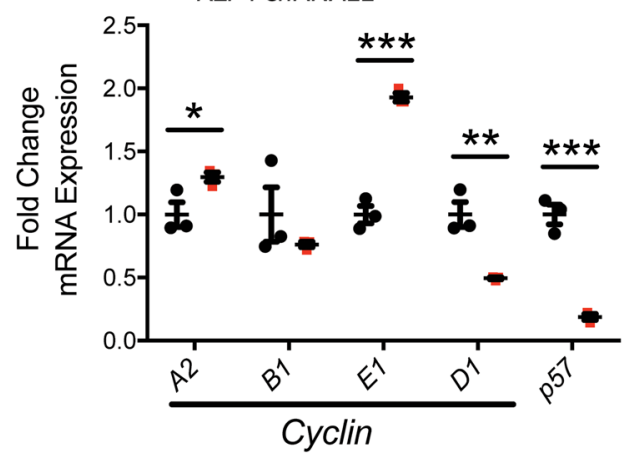

F

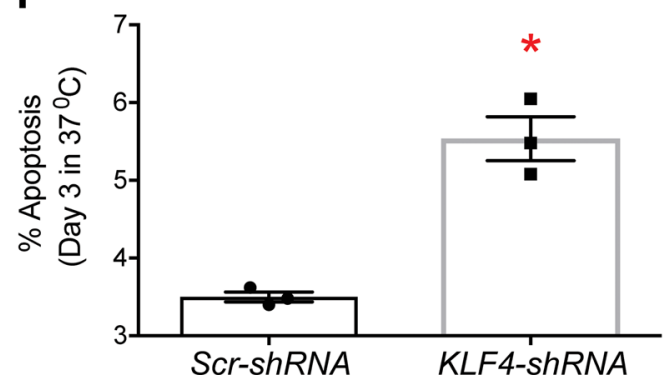

E

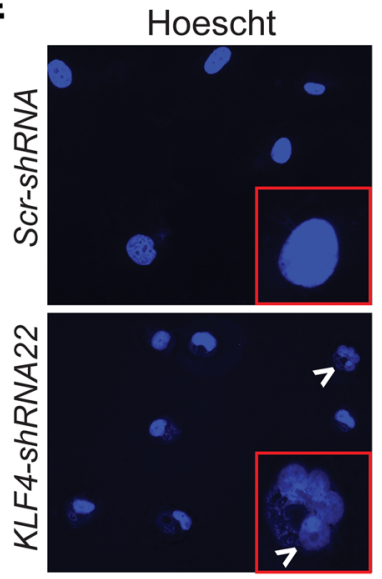

Phalloidin
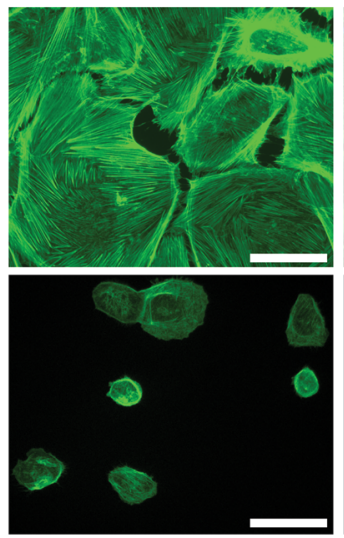

Merged
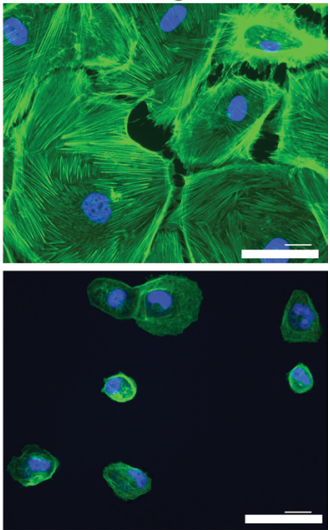

Figure 7. shRNA-mediated KLF4 knockdown increased the susceptibility to mitotic catastrophe. Control (Scr-shRNA) and KLF4 knockdown (KLF4-shRNA21 and KLF4-shRNA22) human podocytes were transferred from $33^{\circ} \mathrm{C}$ (permissive) to $37^{\circ} \mathrm{C}$ (nonpermissive) to induce differentiation.

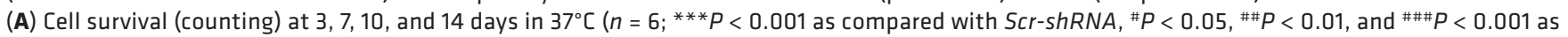
compared with KLF4-shRNA21; 2-way ANOVA with Tukey's multiple comparisons post hoc test). (B) Cell viability (MTS assay) at day 3,7 , and 10 in $37^{\circ} \mathrm{C}\left(n=6-8\right.$; ${ }^{* *} P<0.001$; Mann-Whitney $U$ test). (C) Cell cycle analysis at day 3 in $37^{\circ} \mathrm{C}$. Percentages of human podocytes in sub- $G_{0}, G_{0} / G_{1}, S$, and $G_{2} / M$ phases in left panel $\left(n=3\right.$; ${ }^{*} P<0.05$, ${ }^{* *} P<0.001$; Mann-Whitney $U$ test). Histograms generated with ModFit software to evaluate the relative cell count in each cell cycle stage. (D) CCNA2, CCNB1, CCNE1, CCND1, and $p 57$ mRNA expression are shown relative to Scr-shRNA podocytes at day 3 in $37^{\circ} \mathrm{C}\left(n=3 ;{ }^{*} P<0.05,{ }^{* *} P<0.01,{ }^{* *} P<0.001\right.$; Mann-Whitney $U$ test). (E) Representative images of Phalloidin and Hoechst staining in surviving Scr-shRNA and KLF4-shRNA22 podocytes at 5 days in $37^{\circ} \mathrm{C}$ to evaluate for actin stress fiber formation and apoptotic bodies ( $n=3, \times 20$ ). Inset shows higher magnification $(\times 60)$. Arrowheads show apoptotic bodies. (F) Annexin V/propidium iodide staining in combination with FACS at 3 days in $37^{\circ} \mathrm{C}$ ( $n=3 ;{ }^{*} P<0.05$; Mann-Whitney $U$ test). Scale bars: $100 \mu \mathrm{m}$. 
A

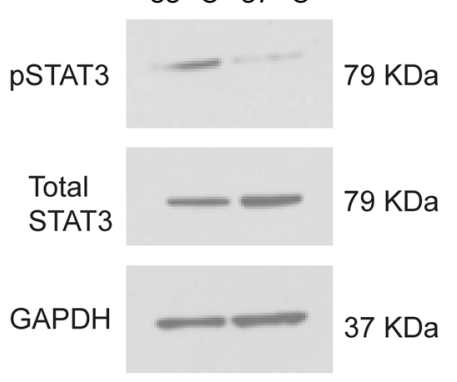

C
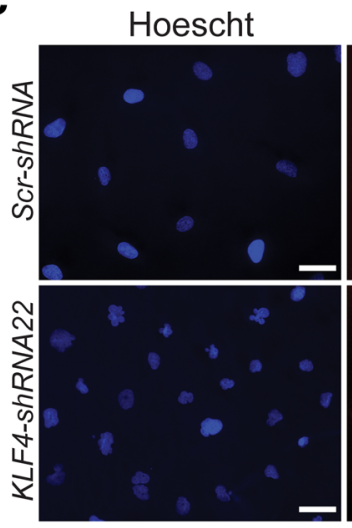

E

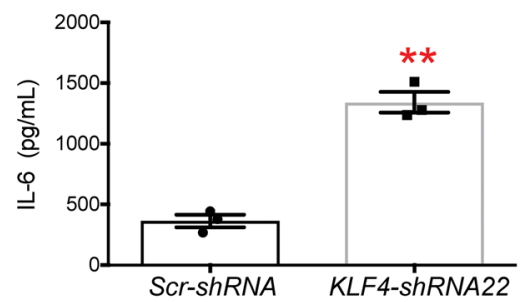

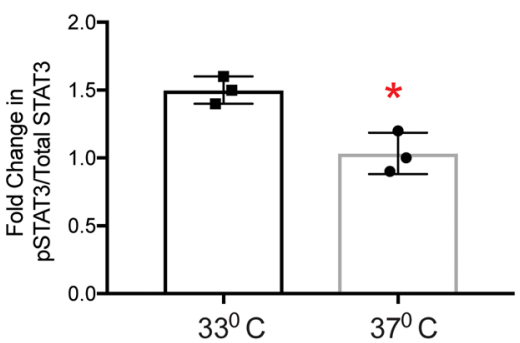

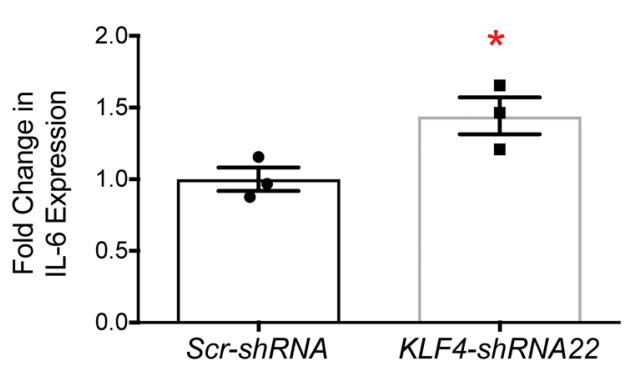

D

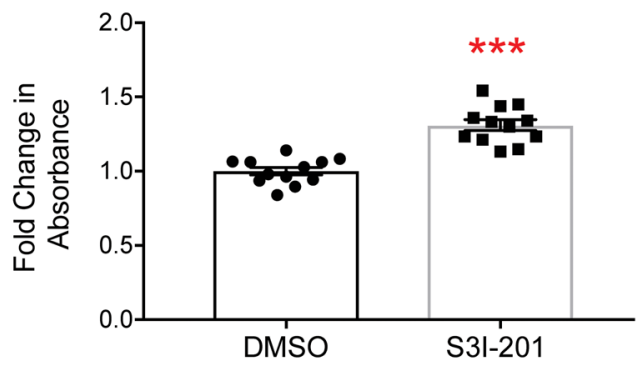

B

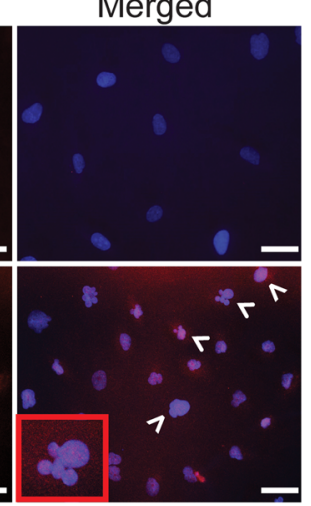

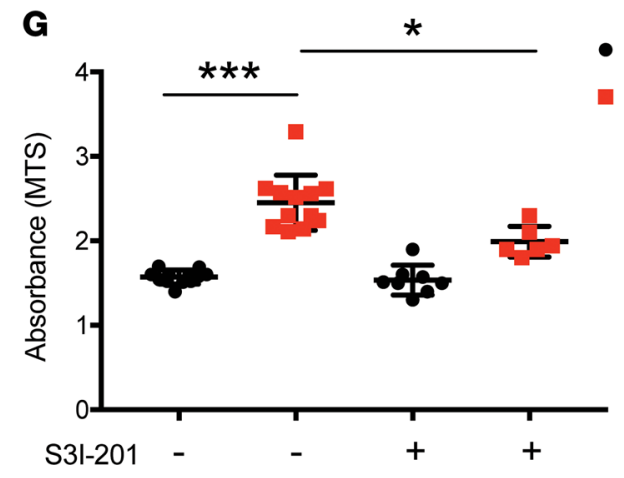

$\mathbf{F}$

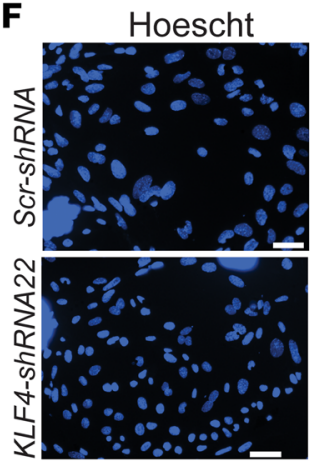

Hoescht
pSTAT3

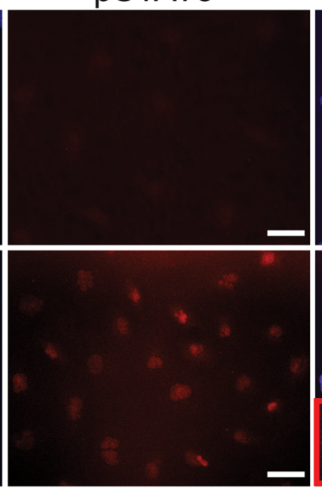

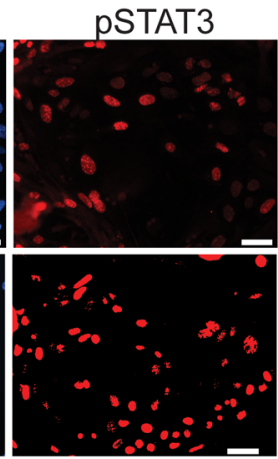

Claudin-1

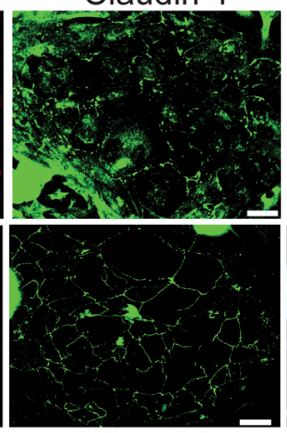

Merged

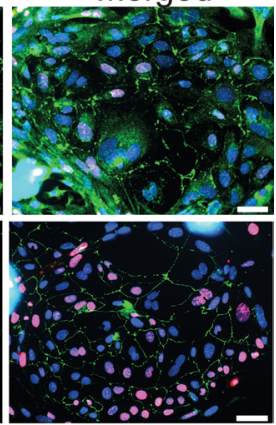

\section{G}

- Scr-shRNA

- KLF4-shRNA22

Figure 8. shRNA-mediated KLF4 knockdown resulted in activation of STAT3 signaling. (A) Representative images of Western blot for phospho-STAT3, total STAT3, and GAPDH in WT human podocytes at $33^{\circ} \mathrm{C}$ and after 14 days of differentiation at $37^{\circ} \mathrm{C}(n=3$, left panel). Quantification of phospho-STAT3 expression by densitometry (right panel) $\left(n=3\right.$; ${ }^{*} P<0.05$; Mann-Whitney $U$ test). (B) IL-6 mRNA expression relative to Scr-shRNA podocytes at day 3 in $37^{\circ} \mathrm{C}\left(n=3\right.$; $^{*} P<0.05$; Mann-Whitney $U$ test). (C) Representative images of phospho-STAT3 and Hoechst immunostaining $(n=3, \times 20)$. Arrowheads show nuclear phospho-STAT3 staining. Inset shown for apoptotic bodies and phospho-STAT3 staining ( $\times 60)$. (D) KLF4-shRNA22 podocytes were treated with S3I-201 $(12.5 \mu \mathrm{m})$ or DMSO for 48 hours under nonpermissive conditions. Cell viability (MTS assay) as a fold change in absorbency $\left(n=10-12\right.$; ${ }^{* *} P<0.001$; Mann-Whitney $U$ test). (E) IL-6 levels in supernatant from KLF4-shRNA22 and Scr-shRNA podocytes at day 3 in $37^{\circ} \mathrm{C}\left(n=3\right.$; ${ }^{*} P<0.01$; Mann-Whitney $U$ test). (F) Glomeruli were isolated from WT mice and cultured for 4 days before treatment with supernatant from Scr-shRNA and KLF4-shRNA22 podocytes for 24 hours. Representative images of Claudin-1 and phospho-STAT3 immunostaining to assess STAT3 activation in PECs $(n=3, \times 20)$. (C) Differentiated mouse PECs treated for 24 hours with supernatant from Scr-shRNA and KLF4-shRNA22 and either S3I-201 or DMSO. Cell proliferation (MTS assay) as a fold change in absorbency $\left(n=10-12 ;{ }^{*} P<0.05,{ }^{* *} P<0.001\right.$; Mann-Whitney $U$ test). Scale bars: $50 \mu \mathrm{m}$. 
A

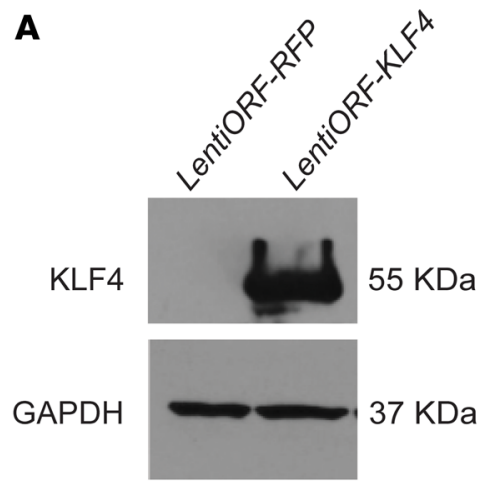

C

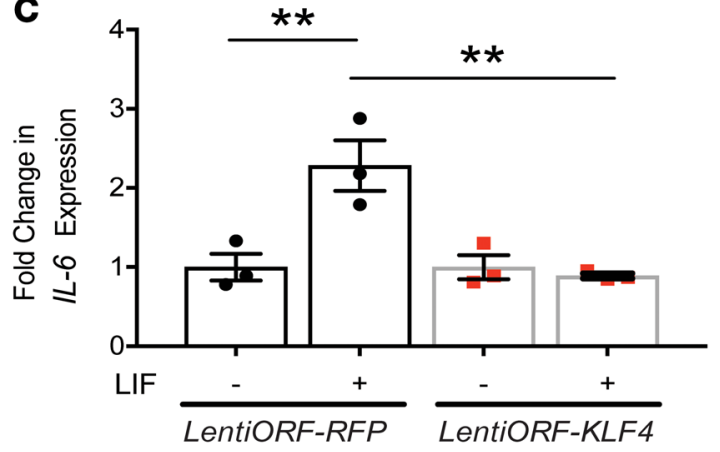

B

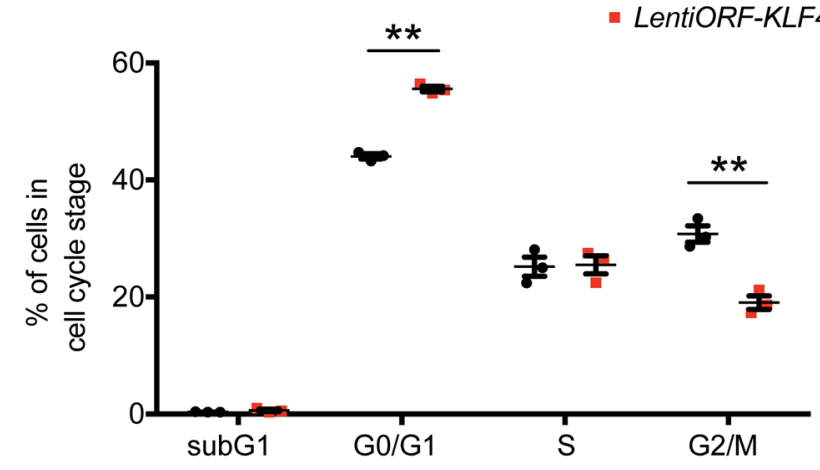

D

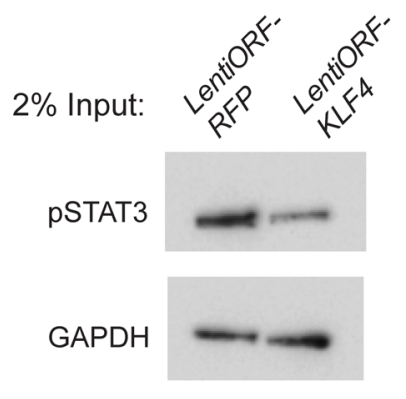

\section{E}

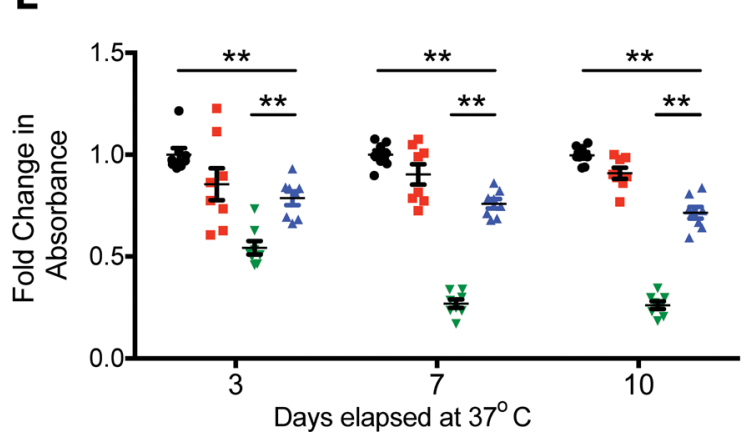

- Lenti-ORF-RFP

- Lenti-ORF-KLF4

- KLF4-shRNA + Lenti-ORF-RFP

$\triangle$ KLF4-ShRNA + Lenti-ORF-KLF4

Days elapsed at $37^{\circ} \mathrm{C}$

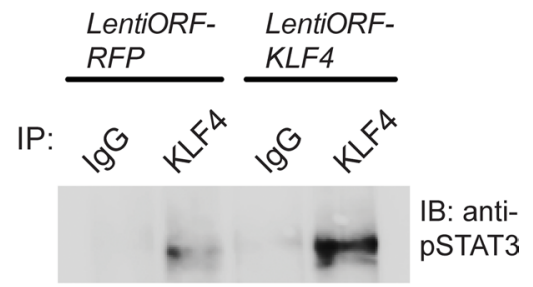

Figure 9. Overexpression of KLF4 reduces cytokine-induced STAT3 signaling and cell cycle progression. (A) Representative Western blot confirms stable overexpression for KLF4 (LentiORF-KLF4) and control vector (LentiORF-RFP) $(n=3)$. (B) Cell cycle analysis of LentiORF-KLF4 and LentiORF-RFP human podocytes under permissive conditions ( $n=3$, ${ }^{* *} P<0.01$; Mann-Whitney $U$ test). (C) Differentiated LentiORF-KLF4 and LentiORF-RFP human podocytes were treated with leukemia inhibitory factor (LIF) (100 ng/ml for 15 minutes) to activate STAT3 signaling, with vehicle (VEH) as control and IL-6 mRNA expression measured ( $n=3$; ${ }^{* *} P<0.01$; Mann-Whitney $U$ test). (D) Representative blot of co-IP with initial IP of KLF4 with anti-KLF4 antibody and immunoblotted for phospho-STAT3 $(n=3)$. IgG isotype serves as control for IP, and GAPDH serves as the loading control. (E) KLF4-shRNA22 podocytes were transfected with LentiORF-KLF4 or LentiORF-RFP, and MTS assay was performed at day 3, 7, and 10 in $37^{\circ} \mathrm{C}\left(n=6-10,{ }^{*} P<0.01\right.$ Kruskal-Wallis test with Dunn's post hoc test).

Increased phospho-STAT3 and decreased KLF4 expression in human RPGN. To evaluate the role of KLF4 in human RPGN, we analyzed previously reported gene expression arrays from micro-dissected glomerular samples from patients with RPGN and healthy living donors deposited in Nephroseq (38), and we observed a significant reduction in glomerular KLF4 expression in RPGN (Figure 10A). To confirm this and to further delineate the relationship between KLF4 and STAT3 signaling in human RPGN, we performed immunostaining for KLF4 and phospho-STAT3 in kidney biopsy specimens from control subjects and from patients with RPGN (including both pauci-immune glomerulonephritis and antiglomerular basement membrane disease). We observed a significant decrease in the percentage of glomerular $\mathrm{KLF}^{+}{ }^{+}$cells with a concomitant increase in the percentage of glomerular phospho-STAT3 ${ }^{+}$cells in RPGN biopsies as compared with control specimens (Figure 10, B and C). This corroborates prior investigations into the role of STAT3 activation in human glomerulonephritis, which showed increased phospho-STAT3 expression in 
A

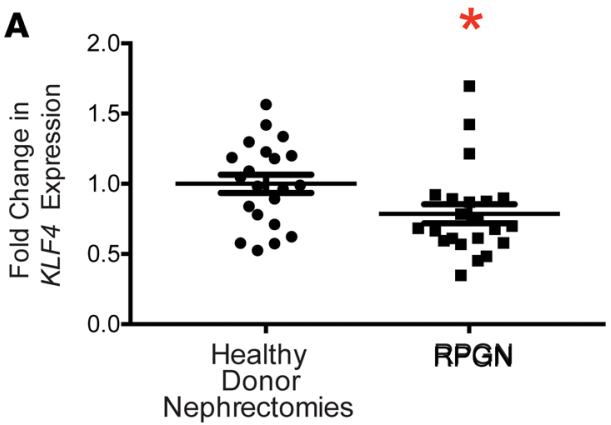

c
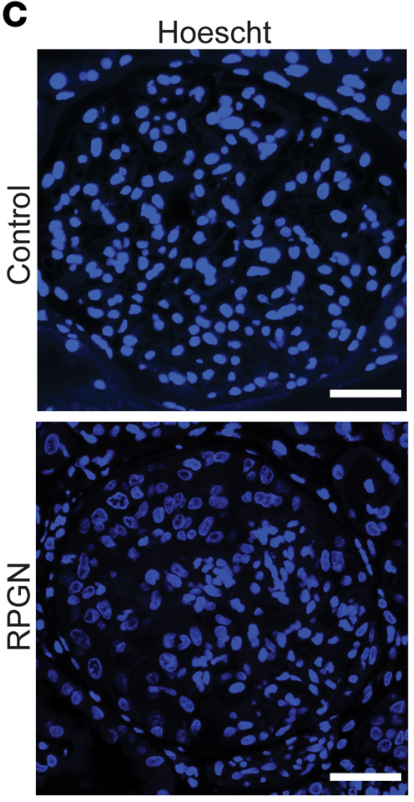
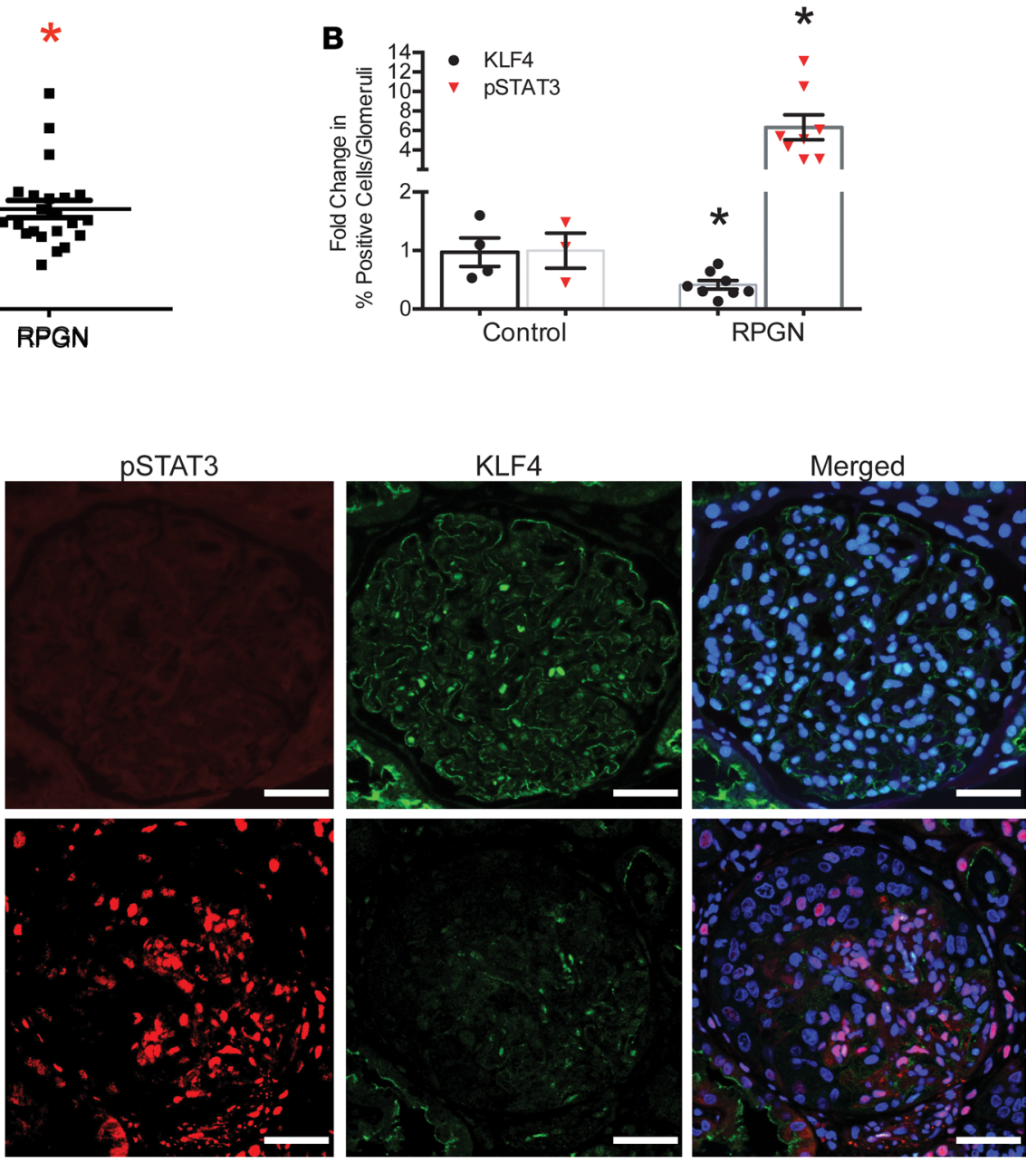

D

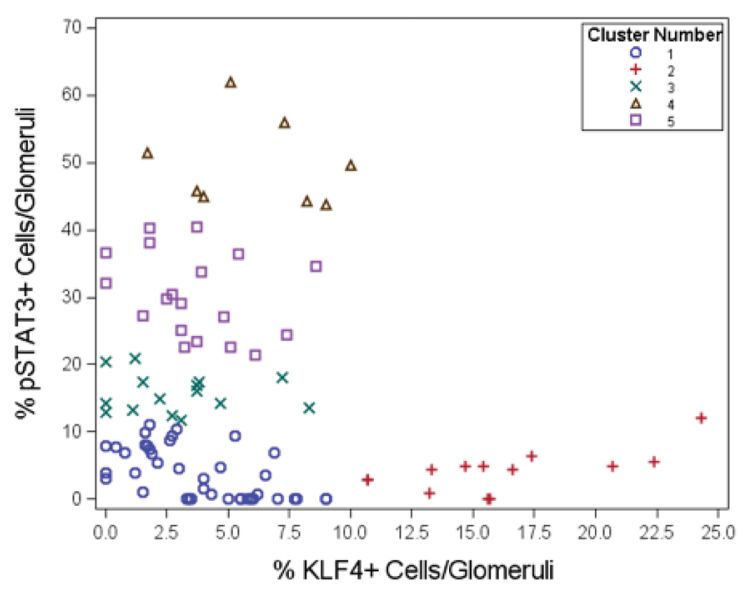

Figure 10. Reduced KLF4 expression and increased phospho-STAT3 expression in human RPGN. (A) Previously reported gene expression from microdissected glomeruli (38) were used to analyze KLF4 mRNA expression from patients with RPGN as compared with normal controls $\left({ }^{*} P<0.05\right.$; unpaired 2-tailed $t$ test). (B) Immunofluorescence for KLF4 and phospho-STAT3 was performed in control $(n=4)$ and RPGN $(n=8)$ specimens. Six to 12 glomeruli per biopsy were selected, and the percentage of KLF4+ and phospho-STAT3 ${ }^{+}$cells were determined by measuring the number of KLF4 and phospho-STAT3 ${ }^{+}$cells relative to total glomerular cells (Hoechst $\left.{ }^{+}\right)\left({ }^{*} P<0.05\right.$ as compared with control; Mann-Whitney $U$ test). (C) The representative images in each group are shown $(\times 20)$. (D) \%KLF4+ and \%phospho-STAT3 ${ }^{+}$in each glomerulus were plotted for healthy donor $(n=36)$ and RPGN biopsies $(n=59)$ and analyzed using hierarchal clustering (Ward's method). With significance set at $P<0.05$, cluster 4 is significantly different from clusters 1 and 2 , cluster 5 is significantly different from clusters 1 and 2 , and cluster 3 is different from cluster 2; the remaining clusters are not significantly different. Scale bars: $50 \mu \mathrm{m}$. 
biopsies of RPGN, with many positive cells located within crescentic lesions (39). We subsequently plotted the expression of KLF4 and phospho-STAT3 in individual glomeruli from all specimens to demonstrate the intraglomeruli variability in staining as well as the inverse relationship between KLF4 and phospho-STAT3 expression within each glomerulus (Figure 10D). Using hierarchal cluster analysis, we observed that glomeruli with high phospho-STAT3 expression ( $>10 \%$ positive cells/glomeruli) formed distinct clusters as compared with those with high KLF4 expression ( $>10 \%$ positive cells/glomeruli), suggesting that the relationship between glomerular phospho-STAT3 and KLF4 expression is considerably altered in RPGN biopsies as compared with control specimens (Figure 10D). Collectively, these data suggest that the loss of KLF4-mediated inhibition of STAT3 signaling might contribute to the pathogenesis of human RPGN.

\section{Discussion}

Here, we identify that KLF4/STAT3 signaling in the podocyte has an essential role in the initiation and progression of aberrant GEC hyperplasia in murine models of RPGN and FSGS. We illustrated this by demonstrating that the genetic knockdown of Klf4 specifically in the podocytes in mice on C57BL/6J background increased their susceptibility to NTS nephritis. Furthermore, podocyte-specific knockdown of Klf4 was sufficient to induce FSGS with aberrant GEC proliferation, renal failure, and a significant reduction in overall survival on the FVB/N background. Klf4 knockdown in both models was accompanied by STAT3 activation and pathologic GEC proliferation. Moreover, in differentiated podocytes, KLF4 is critical to the maintenance of mature podocyte differentiation markers, cell cycle senescence, and preventing cell death; its loss is associated with STAT3 activation and subsequent IL-6 secretion, which is a potential mediator of podocyte-PEC crosstalk. Importantly, administration of a selective STAT3 inhibitor partially mitigated these effects both in our murine model and in vitro. Finally, we observed a significant reduction in KLF4 expression with a reciprocal increase in phospho-STAT3 expression in glomeruli from kidney biopsies with RPGN as compared with control specimens.

In light of the reported role of KLF4 as a negative regulator of the cell cycle (15), its vital function in the maintenance of mature differentiated podocyte markers (actin stress fiber formation and cell cycle arrest) is not surprising. Developing podocytes express Cyclin A, Cyclin B1, and Ki67 until the capillary loop stage; thereafter, there is no further expression of these markers, coinciding with increasing expression of Cyclin D1, p27, p57, and Synaptopodin (40). Furthermore, the persistent activation of these markers is required for quiescence and $\mathrm{G}_{0}$ phase arrest in mature terminally differentiated podocytes (40-42). Here, we show that KLF4 knockdown in cultured podocytes is accompanied by the loss of Cyclin D1 and p57 expression with an increase in Cyclin $A 2$ and $-E 1$ expression, as well as a shift from $\mathrm{G}_{0} / \mathrm{G}_{1}$ to the $\mathrm{S}$ and $\mathrm{G}_{2} / \mathrm{M}$ phases. Importantly, this cell cycle reentry was associated with a significant reduction in cell survival. Similarly, mice with podocyte-specific Klf4 deletion (FVB/N background) also exhibited a reduction in p57 expression, as well as an increase in mitotic and apoptotic GECs. These observations are in accordance with prior studies that showed similar disruptions of cell-cycle regulatory proteins in podocytopathies, such as increased expression of Cyclin B1 and -B2 in murine models of HIVAN (43), Cyclin A in human biopsies of collapsing glomerulopathy (44), and Cyclin E in cellular FSGS (30). Previous reports have demonstrated that KLF4 transcriptionally represses Cyclin $E$ (17) and activates p57 expression (15, 35), supporting our findings that the loss of KLF4 in differentiated podocytes is associated with dysregulated cell-cycle reentry. Here, we also show that this cell cycle reentry was accompanied by destabilization of actin cytoskeleton and apoptosis. This combination of aberrant podocyte mitosis and cell death, termed mitotic catastrophe, was previously reported, where differentiated human podocytes treated with mitogenic stimuli had increased susceptibility to apoptosis (45). Furthermore, podocytes undergoing mitotic catastrophe, as evidence of pathologic cell cycle reentry and subsequent loss, has been observed in human biopsies of IgA nephropathy and collapsing FSGS (46).

Alterations in cell cycle regulatory proteins alone are unlikely to be sufficient to initiate podocyte injury and/or loss. This has been demonstrated in prior studies with $p 57-\mathrm{KO}$ mice that have normal glomerular development (42), as well as by our finding that mice with podocyte-specific Klf4 knockdown on C57BL/6J background did not develop overt disease. Instead, we postulate that enhanced activation of STAT3 signaling is required for the loss of mature differentiation markers and eventual podocyte loss. STAT3 activation in the podocyte has been demonstrated to be pathologic in both the HIVAN (47) and NTS (6) murine models. In keeping with this, our observations revealed a reduction in STAT3 activation in podocytes under nonpermissive, differentiated conditions as compared with permissive conditions. Interestingly, the 
loss of KLF4 reactivated STAT3 signaling under nonpermissive conditions, leading to mitotic catastrophe and podocyte death while triggering IL-6-induced paracrine signaling between podocytes and PECs. However, our studies demonstrate that it is possible to suppress this aberrant reactivation of STAT3 signaling in KLF4-knockdown podocytes by either restoring KLF4 expression or with pharmacological inhibition of STAT3 signaling. Our data is supported by recent publications by Qin et al. delineating the interaction between KLF4 and STAT3 in neurons, where KLF4 binds to phosphorylated STAT3 and suppresses its downstream signaling (21) and by our co-IP assays demonstrating the similar interaction exists in podocytes. Moreover, this inhibitory interaction, combined with studies that have shown KLF4 as a downstream STAT3 target $(23,48,49)$, is suggestive of a novel negative-feedback pathway in podocytes.

Of particular interest is the strain-dependent nature of glomerular STAT3 expression after Klf4 knockdown. Here, we observed that the podocyte-specific loss of Klf4 on the FVB/N background was sufficient to render the development of FSGS with podocyte detachment and GEC proliferation, which might be due to enhanced constitutive STAT3 activation observed in this strain. Consistent with this, previous studies demonstrated that STAT3 activation drives chronic kidney disease progression preferentially in FVB/N as compared with C57BL/6J mice, with transcriptome profiling revealing an enrichment in STAT3 conserved binding sites in the genes differentially expressed in the FVB/N mice (29). This might speak to why, as demonstrated by Hayashi et al, decreased glomerular Klf4 expression is not sufficient to drive PEC proliferation in either the Adriamycin nephropathy or diabetic nephropathy murine models on a C57BL/6J background (19). It is also likely that the consequences of reduced podocyte-specific Klf4 are dose dependent in addition to strain dependent. In keeping with this, the phenotypic diversity observed between our C57BL/6J and FVB/N strains might also be due to variable efficiency of Klf4 knockdown, perhaps secondary to the mosaicism of $C r e$ recombinase activity. These effects can be mitigated in future studies by using CRISPR-Cas9 technology.

Pharmacologic inhibition of STAT3 activation with S3I-201 partially restored survival in KLF4-knockdown podocytes in nonpermissive conditions and attenuated albuminuria in Podocin-Cre Klf4//fl mice on the FVB/N background. The vast majority of work regarding STAT3 inhibition in kidney disease has been done in fibrosis, where STAT3 inhibition with administration of S3I-201 (34), paclitaxel (50), or mesenchymal stem cells (51) has reduced fibroblast activation and attenuated fibrosis after unilateral ureteric obstruction in mice. In glomerular disease, administration of the small molecule inhibitor of STAT3, Stattic, delayed onset of lupus nephritis in a murine model (52). Collectively, these data raise the possibility of STAT3 inhibition as a viable therapeutic target in glomerular diseases such as RPGN.

Although podocytes may reenter the cell cycle following injury or mitogenic stimuli, recent studies have demonstrated that they do not comprise the majority of the proliferating GECs in proliferative glomerulonephritis (GN) $(2,3)$. While we cannot definitively conclude the source of the GECs without lineage tracing, we hypothesize that STAT3-mediated mitotic catastrophe in the podocyte stimulates aberrant PEC proliferation in this model. In fact, this podocyte-PEC cross-talk has been implicated previously by Dai et al., where podocyte-specific deletion of Stat 3 reduced PEC accumulation in NTS nephritis (6). While STAT3 activation is traditionally antiapoptotic and induces cellular proliferation (53), this is likely cell-context dependent (54). We suggest STAT3 is proapoptotic in podocytes, as their proliferative capacity is limited by their $\mathrm{G}_{0}$ arrest (45), and paracrine signaling between the injured podocyte and PECs is likely responsible for PEC accumulation. A potential mediator of cell-cell communication between podocytes and PECs is IL-6, which is both a STAT3 activator and a downstream target (55). Previous studies have shown that podocytes both express and secrete IL-6 in response to inflammatory mediators such as LPS (56) or TNF- $\alpha$ (57), and circulating IL-6 levels are significantly increased in patients with RPGN (58). Our data corroborates these observations and confirms that the loss of KLF4 in differentiated podocytes in culture induces IL- 6 secretion during differentiation. Furthermore, treatment of PECs with this IL-6-containing supernatant is sufficient to trigger STAT3 activation and proliferation. Future studies will focus in more detail on the effect of podocyte-specific IL-6 induction on PECs, as well as identification of additional STAT3-induced secreted molecules that might mediate the cross-talk between podocytes and PECs.

The prodifferentiation and salutary effects of KLF4 in the podocyte might overlap with other members in the KLF family. For instance, we previously showed that KLF15 transcriptionally regulates podocyte differentiation genes (13) and is required for the beneficial effects of glucocorticoids in the podocyte after injury (59). Furthermore, prior studies also revealed that glomerular expression of Klf15 was increased in mice 
with podocyte-specific Klf4 deletion on the C57BL/6J background (19), suggesting a potential compensatory response to prevent podocyte injury at baseline in this strain. However, it remains unclear whether this compensatory response is lost in the FVB/N strain or in KLF4-knockdown podocytes, thereby contributing to the injury observed in these models. Furthermore, the reno-protective role of KLF4 might be attributed to the demethylation of the Nephrin promoter with enhanced promoter activity (19). However, after podocyte-specific Klf4 knockdown, these epigenetic changes were only reported to occur in the setting of podocyte stress (19); therefore, it remains unclear if it might contribute to phenotypic changes observed in both differentiated human podocytes and in FVB/N mice with KLF4 knockdown. These epigenetic changes mediated by KLF4 will need to be addressed in subsequent studies by examining the reno-protective role of podocyte-specific KLF4 induction in murine models of proliferative GN.

Finally, immunostaining of human biopsy specimens confirmed the nuclear and cytosolic distribution of KLF4 previously observed, as well as its primarily glomerular localization (19). Importantly, the dysregulated relationship between glomerular KLF4 and phospho-STAT3 expression in RPGN biopsy specimens supports our claim regarding the essential role of this signaling pathway in proliferative glomerulonephritis. Whether the ratio of KLF4/phospho-STAT3 expression correlates with disease severity, progression, or response to therapy remains uncertain and will be the basis for further investigations.

Taken together, these data provide evidence toward the essential role of KLF4/STAT3 signaling in the podocyte in proliferative glomerular lesions, which, to the best of our knowledge, has not been previously described. Specifically, our studies suggest that podocyte-specific KLF4 is required for the mature podocyte phenotype, including cell cycle quiescence, and its downregulation results in the activation of STAT3 signaling with a subsequent loss of differentiation markers and reduced survival. This is further supported by the reciprocal pattern of KLF4 and phospho-STAT3 expression in RPGN as compared with control kidney biopsy specimens. Furthermore, our data provide a glimpse toward potential mediators of podocyte/PEC paracrine signaling, which will be the basis for future studies. In all, enhancing KLF4-mediated inhibition of STAT3 signaling might serve as a viable target for therapy in glomerulopathies with aberrant GEC proliferation.

\section{Methods}

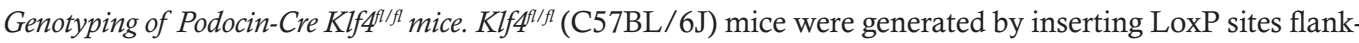
ing exons 2 and 3 of the Klf4 gene as previously reported (60). Klf4t/fl mice (C57BL/6J) were crossed with mice expressing Cre recombinase under the control of the Podocin promoter (B6.Cg-Tg [NPHS2-cre] 295Lbh/J; The Jackson Laboratory). Klf4 $4^{l / f l}$ mice were crossed 9 generations with Podocin-Cre (FVB/N) to generate Podocin-Cre Klf4/f/fl $(\mathrm{FVB} / \mathrm{N})$ mice. Genotyping was performed as previously described (61).

NTS nephritis model. In the NTS model, 12-week old Podocin-Cre Klf4//fl and Podocin-Cre Klf4 ${ }^{+/+}$male littermates were first sensitized with an i.p. injection of $0.5 \mathrm{mg}$ sheep IgG (Jackson ImmunoResearch) with complete Freund's adjuvant (MilliporeSigma) or PBS. Five days later, mice were administered either 100 $\mu 1$ of NTS or VEH (sterile PBS), i.p. Urine was collected prior to IgG injection and after 7 and 14 days. Serum was collected prior to injection and the time of sacrifice. Mice were euthanized at either day 7 or 14 after injection, and kidneys were harvested for histology, protein, RNA, and glomerular isolation. Urine albumin was quantified by ELISA (Bethyl Laboratory Inc.). Urine creatinine levels were measured in the same samples using the QuantiChrom Creatinine Assay Kit (DICT-500; BioAssay Systems) according to the manufacturer's instructions. Serum creatinine was measured by the Isotope Dilution LC-MS/MS at the University of Alabama at Birmingham O'Brien Core Center.

S3I-201 treatment protocol in mice. Podocin-Cre Klf4/flfl $(\mathrm{FVB} / \mathrm{N})$ mice with dipstick-positive proteinuria, at 7 weeks of age, were randomized to receive either S3I-201 $10 \mathrm{mg} / \mathrm{kg}$ (Santa Cruz Biotechnology Inc.) or DMSO, i.p., 3 times weekly according to previously published protocols $(34,62,63)$. Urine was collected weekly up until 11 weeks of age.

Isolation of glomeruli, primary podocytes, and PECs from mice. Mouse glomeruli were isolated as previously described (64). After glomerular isolation, primary mouse podocytes were isolated as previously described (65) and processed for RNA or protein preparation. For PEC isolation, glomeruli were cultured on collagen 1-coated slide plates in RPMI 1640 (Gibco) containing 10\% FBS (Cansera International) supplemented with $100 \mathrm{U} / \mathrm{ml}$ penicillin. Cultures were incubated in a $37^{\circ} \mathrm{C}$ humidified incubator for 4 days prior to treatment with $10 \%$ supernatant from podocytes with KLF4-knockdown or controls. PEC outgrowths from capsulated glomeruli were confirmed by Claudin-1 staining. 
Cell culture. Conditionally immortalized human podocytes were previously a gift from Peter Mundel (Massachusetts General Hospital, Boston, Massachusetts, USA). Methods for podocyte cultivation, immortalization, and differentiation were based on a previously described protocol (59).

KLF4 knockdown in podocytes was performed using Genecopoeia lentiviral shRNAmir system with HSH022519-21-LVRU6GP (KLF4-shRNA21) and HSH022519-22-LVRU6GP (KLF4-shRNA22) and CSHCTR001-LVRU6GP (Scr-shRNA) constructs. LentiORF-KLF4 clone was purchased from Genecopoeia, and $K L F 4$ overexpression was achieved by transfecting human podocytes using Viafect (Promega). Cells were selected with $1.25 \mu \mathrm{g} / \mathrm{ml}$ puromycin for 2-3 weeks prior to use in all studies. Western blot was performed to confirm KLF4 overexpression in LentiORF-KLF4 as compared with LentiORF-RFP podocytes. For IP studies, cells were lysed with radioimmunoprecipitation assay buffer with protease inhibitors and immunoprecipitated with antibodies against rabbit anti-KLF4 antibody (PM057, MBL). Interacting phospho-STAT3 was detected by immunoblotting with rabbit anti-phospho-STAT3 antibody (D3A7, Cell Signaling Technologies). As controls, protein lysates transfected with the control vector (LentiORF-RFP) and precipitation with the IgG isotype control were used for each group. Input (2\%) of whole cell lysates was immunoblotted with phospho-STAT3 and GAPDH to detect protein expression.

Immortalized mouse PECs were a gift from Stuart Shankland (University of Washington Medical Center, Seattle, Washington, USA). Methods for cultivation and differentiation of mouse PECs were based on previously described protocols $(66,67)$. Cells were initially cultured under growth-permissive conditions at $33^{\circ} \mathrm{C}$ and then differentiated at $37^{\circ} \mathrm{C}$ for 14 days prior to being used for experiments.

For studies involving cell counting, we followed the manufacturer's protocol using the Z1 Coulter Particle Counter (Beckman Coulter). Cells were evaluated for apoptosis using a FACS Calibur flow cytometer as previously reported (61). For LIF experiments, human podocytes were seeded in 6-well plates at the confluence of $70 \%$, placed in $37^{\circ} \mathrm{C}$ for 14 days, and subsequently treated with LIF (100 $\mathrm{ng} / \mathrm{ml}$ ) or VEH for 15 minutes. Subsequently, cells were harvested for real-time PCR and Western blot. For S3I-201 experiments, human podocytes (KLF4-shRNA22) were seeded in 96-well plates at 2,000 cells per well and treated with S3I-201 (Santa Cruz Biotechnology Inc.) or DMSO at $12.5 \mu \mathrm{m}$ starting at day 1 under nonpermissive conditions. Media containing S3I-201 was replaced at 48 hours, and MTS assay was performed. Cell cycle analysis was performed on an FACS Calibur Flow Cytometer at Stony Brook University with data analysis as previously described (59). To assess cell proliferation, MTS solution (Promega) was added to the culture media, and plates were incubated for 2 hours at $37^{\circ} \mathrm{C}$ with $5 \% \mathrm{CO}_{2}$. Optical density was determined at $490 \mathrm{~nm}$ using a 96 -well plate reader SpectraMax M3 (Molecular Devices).

IL-6 measurement in supernatant. ELISA (Cayman Chemicals) for human IL-6 was performed on the supernatant from human podocytes with and without KLF4 knockdown (Scr-shRNA and KLF4-shR$N A 22$ ). Cells were seeded at 600,000 per $15-\mathrm{cm}$ plate and placed in $37^{\circ} \mathrm{C}$ to differentiate. After 24 hours, media was removed and cells were washed 5 times with PBS. Phenol-red, ITS, and serum-free RPMI was replaced. After 48 additional hours, supernatant was harvested and centrifuged for 5 minutes at 500 $\mathrm{g}$ and then for 10 minutes at $1,500 \mathrm{~g}$ before being filtered through a 200 -nm filter and stored in $-80^{\circ} \mathrm{C}$ prior to ELISA.

Real-time PCR. Total RNA was extracted from cells by using TRIzol (Gibco) or from glomeruli of mice using the RNeasy Kit (Qiagen). CDNA preparation and real-time PCR was performed as previously described (59). Primer sequences for mouse Klf4, mouse Il-6, mouse Socs3, mouse Stat3, mouse Icam-1, mouse Synpo, mouse Nephrin, mouse Wt1, human KLF4, human IL-6, and human $p 57$ were designed using NCBI/Primer-BLAST (Supplemental Table 1). Primers for CCNA2 (qt00014798), CCNB1 (qt0000615), CCNE1 (qt00041986) and CCND1 (qt00495285) were purchased from Qiagen.

Western blot analysis. Western Blot analysis using rabbit anti-KLF4 (PM057; MBL), rabbit anti-GAPDH (MAB374; MilliporeSigma), mouse anti-total-STAT3 (124H6; Cell Signaling Technologies), and rabbit anti-phospho-STAT3 (D3A7; Cell Signaling Technologies) was done as previously reported (61). Blots were either developed as previously reported (61) or with the Azure c400 Gel Imaging System.

Histopathology. Mice were perfused with PBS, and the kidneys were fixed, embedded, and sectioned as previously described (61). Slides were stained with PAS (MilliporeSigma), H\&E, and Masson's trichrome staining. Quantification for both C57BL/6J and FVB/N mice was performed, blinded, by renal pathologist (M.P. Revelo) using the following scoring system for inflammation and fibrosis: 0 , none; $1,<10 \% ; 2$, $10 \%-25 \% ; 3,>25 \%-50 \%$. 
Transmission EM was performed as recently described (61) using a Hitachi H7650 Microscope. Negatives were digitalized, and images with a final magnitude of approximately $\times 6,800, \times 13,000$, and $\times 49,000$ were obtained.

Immunofluorescence. Immunofluorescence for goat anti-KLF4 antibody (AF3158; R\&D Systems), rabbit anti-phospho-STAT3 antibody (D3A7; Cell Signaling), rabbit anti-Ki67 antibody (CRM325B; Biocare Medical), mouse anti-Claudin-1 (81796; Santa Cruz Biotechnology Inc.), rabbit anti-Nephrin (NBP1-30130; Novus), mouse anti-WT1 (sc-7385 Santa Cruz Biotechnology Inc.), rat anti-Nestin (MAB353; MilliporeSigma), mouse anti-p57 (sc-56456; Santa Cruz Biotechnology Inc.), goat anti-Synaptopodin (sc-21537; Santa Cruz Biotechnology Inc.), or rat anti-CD44 (103001; BioLegend) was performed as recently reported (61). After staining, slides were photographed under a Nikon Eclipse i90 microscope with a digital camera. Quantification of intensity and cell number was performed using ImageJ 1.26t software (NIH, rsb.info.nih.gov/ij).

Deidentified human kidney biopsy specimens with RPGN from Stony Brook University and University of Utah were obtained for staining of KLF4 and phopsho-STAT3. The diagnosis of RPGN in kidney biopsy specimens was provided by the renal pathologist (M.P. Revelo). Control kidney biopsy specimens were acquired from the unaffected pole of nephrectomies. Percentage of glomerular KLF4 and phospho-STAT3 cells were quantified by counting the total number of either KLF4 or phospho-STAT3 ${ }^{+}$cells and dividing over total number of Hoechst ${ }^{+}$cells.

Statistics. All continuous data was assessed for normality, and then parametric or nonparametric tests were employed for data analysis, as appropriate. For normally distributed data, a 2-tailed $t$ test was used to compare data between 2 groups, and 1-way ANOVA with Tukey's post hoc test was used to compare data between more than 2 groups. For data sets in which we could not assume normality, nonparametric statistical tests were performed using the Mann-Whitney $U$ test to compare data between 2 groups and the Kruskal-Wallis test with Dunn's post hoc test to compare data between more than 2 groups. Continuous data are expressed as mean \pm SEM. All experiments were repeated a minimum of 3 times, and representative experiments are shown. Kaplan-Meier survival analysis with log-rank test was used to generate and compare survival curves in mice. Statistical significance was considered when $P<0.05$, and analysis was performed using GraphPad Prism 6.0a. A hierarchal cluster analysis (Ward's method) was conducted in SAS v9.4 and was performed to analyze the relationship between KLF4 and phospho-STAT3 in individual glomeruli from patients with RPGN versus controls.

Study approval. All animal studies conducted were approved by the Stony Brook University Animal Institute Committee. Stony Brook University IRB approved the use of archived deidentified human biopsy specimens for immunostaining.

\section{Author contributions}

CCE, SKM, PP, SC, and YG designed experiments. CCE and SKM wrote the draft of the manuscript, followed by editing by VWY, AM, SJS, JCH, VDD, DJS, and ABB. CCE, PP, SC, JP, and YG performed all experiments. MPR and VDD quantified all histology and/or interpreted histological findings. NTS was provided by DJS.

\section{Acknowledgments}

This work was supported by funds from the NIH/NIDDK (DK102519 and DK112984 to SKM and DK112618 to CCE), VA Merit (1I01BX003698 to SKM), AHA Grant-In-Aid (16GRNT31280004 to SKM), and Dialysis Clinic Inc. (Paul Teschan Research Grant to CCE and SKM). We thank Erin Taub for statistical support.

Address correspondence to: Sandeep K. Mallipattu, Department of Medicine/Nephrology, Stony Brook University, 100 Nicolls Road, HSCT17-090B, Stony Brook, New York, USA. Phone: 631.638.2164; Email: sandeep.mallipattu@stonybrookmedicine.edu.

\footnotetext{
1. D'Agati VD, Fogo AB, Bruijn JA, Jennette JC. Pathologic classification of focal segmental glomerulosclerosis: a working proposal. Am J Kidney Dis. 2004;43(2):368-382.

2. Smeets B, et al. Tracing the origin of glomerular extracapillary lesions from parietal epithelial cells. J Am Soc Nephrol. 2009;20(12):2604-2615.
} 
3. Moeller MJ, et al. Podocytes populate cellular crescents in a murine model of inflammatory glomerulonephritis. $J$ Am Soc Nephrol. 2004;15(1):61-67.

4. Shankland SJ, Anders HJ, Romagnani P. Glomerular parietal epithelial cells in kidney physiology, pathology, and repair. Curr Opin Nephrol Hypertens. 2013;22(3):302-309.

5. Ding M, et al. Loss of the tumor suppressor Vhlh leads to upregulation of Cxcr4 and rapidly progressive glomerulonephritis in mice. Nat Med. 2006;12(9):1081-1087.

6. Dai Y, et al. Podocyte-specific deletion of signal transducer and activator of transcription 3 attenuates nephrotoxic serum-induced glomerulonephritis. Kidney Int. 2013;84(5):950-961.

7. Feng X, et al. Reduction of Stat3 activity attenuates HIV-induced kidney injury. J Am Soc Nephrol. 2009;20(10):2138-2146

8. Verstovsek S, et al. Safety and efficacy of INCB018424, a JAK1 and JAK2 inhibitor, in myelofibrosis. N Engl J Med. 2010;363(12):1117-1127.

9. Bergrath E, Gerber RA, Gruben D, Lukic T, Makin C, Wallenstein G. Tofacitinib versus Biologic Treatments in Moderate-to-Severe Rheumatoid Arthritis Patients Who Have Had an Inadequate Response to Nonbiologic DMARDs: Systematic Literature Review and Network Meta-Analysis. Int J Rheumatol. 2017;2017:8417249.

10. Bialkowska AB, Yang VW, Mallipattu SK. Krüppel-like factors in mammalian stem cells and development. Development. 2017;144(5):737-754

11. Mallipattu SK, Estrada CC, He JC. The critical role of Krüppel-like factors in kidney disease. Am J Physiol Renal Physiol. 2017;312(2):F259-F265.

12. McConnell BB, Yang VW. Mammalian Krüppel-like factors in health and diseases. Physiol Rev. 2010;90(4):1337-1381

13. Mallipattu SK, et al. Kruppel-like factor 15 (KLF15) is a key regulator of podocyte differentiation. J Biol Chem. 2012;287(23):19122-19135.

14. Shields JM, Christy RJ, Yang VW. Identification and characterization of a gene encoding a gut-enriched Krüppel-like factor expressed during growth arrest. J Biol Chem. 1996;271(33):20009-20017.

15. Chen X, Whitney EM, Gao SY, Yang VW. Transcriptional profiling of Krüppel-like factor 4 reveals a function in cell cycle regulation and epithelial differentiation. J Mol Biol. 2003;326(3):665-677.

16. Jeon GI, Yoon MY, Park HR, Lee SC, Park E. Neuroprotective activity of Viola mandshurica extracts on hydrogen peroxide-induced DNA damage and cell death in PC12 cells. Ann N Y Acad Sci. 2009;1171:576-582.

17. Yoon HS, Ghaleb AM, Nandan MO, Hisamuddin IM, Dalton WB, Yang VW. Krüppel-like factor 4 prevents centrosome amplification following gamma-irradiation-induced DNA damage. Oncogene. 2005;24(25):4017-4025.

18. Yoon HS, Yang VW. Requirement of Krüppel-like factor 4 in preventing entry into mitosis following DNA damage. J Biol Chem. 2004;279(6):5035-5041.

19. Hayashi K, et al. KLF4-dependent epigenetic remodeling modulates podocyte phenotypes and attenuates proteinuria. $J$ Clin Invest. 2014;124(6):2523-2537.

20. Chen X, et al. Krüppel-like factor 4 (gut-enriched Krüppel-like factor) inhibits cell proliferation by blocking G1/S progression of the cell cycle. J Biol Chem. 2001;276(32):30423-30428.

21. Qin S, Zou Y, Zhang CL. Cross-talk between KLF4 and STAT3 regulates axon regeneration. Nat Commun. $2013 ; 4: 2633$.

22. Cui DM, et al. KLF4 Knockdown Attenuates TBI-Induced Neuronal Damage through p53 and JAK-STAT3 Signaling. CNS Neurosci Ther. 2017;23(2):106-118.

23. Niwa H, Ogawa K, Shimosato D, Adachi K. A parallel circuit of LIF signalling pathways maintains pluripotency of mouse ES cells. Nature. 2009;460(7251):118-122.

24. Hall J, et al. Oct4 and LIF/Stat3 additively induce Krüppel factors to sustain embryonic stem cell self-renewal. Cell Stem Cell. 2009;5(6):597-609.

25. Yoshida T, Yamashita M, Iwai M, Hayashi M. Endothelial Krüppel-Like Factor 4 Mediates the Protective Effect of Statins against Ischemic AKI. J Am Soc Nephrol. 2016;27(5):1379-1388

26. Hayashi K, et al. Renin-angiotensin blockade resets podocyte epigenome through Kruppel-like Factor 4 and attenuates proteinuria. Kidney Int. 2015;88(4):745-753.

27. Salant DJ, Cybulsky AV. Experimental glomerulonephritis. Meth Enzymol. 1988;162:421-461.

28. Laouari D, et al. TGF-alpha mediates genetic susceptibility to chronic kidney disease. J Am Soc Nephrol. 2011;22(2):327-335

29. Bienaimé F, et al. Stat3 Controls Tubulointerstitial Communication during CKD. J Am Soc Nephrol. 2016;27(12):3690-3705.

30. Wang S, Kim JH, Moon KC, Hong HK, Lee HS. Cell-cycle mechanisms involved in podocyte proliferation in cellular lesion of focal segmental glomerulosclerosis. Am J Kidney Dis. 2004;43(1):19-27.

31. He JC, et al. Nef stimulates proliferation of glomerular podocytes through activation of Src-dependent Stat 3 and MAPK1,2 pathways. J Clin Invest. 2004;114(5):643-651.

32. Sharma M, et al. Janus kinase 2/signal transducer and activator of transcription 3 inhibitors attenuate the effect of cardiotrophin-like cytokine factor 1 and human focal segmental glomerulosclerosis serum on glomerular filtration barrier. Transl Res. 2015;166(4):384-398.

33. Siddiquee K, et al. Selective chemical probe inhibitor of Stat3, identified through structure-based virtual screening, induces antitumor activity. Proc Natl Acad Sci USA. 2007;104(18):7391-7396

34. Pang M, et al. A novel STAT3 inhibitor, S3I-201, attenuates renal interstitial fibroblast activation and interstitial fibrosis in obstructive nephropathy. Kidney Int. 2010;78(3):257-268.

35. Ky N, Lim CB, Li J, Tam JP, Hamza MS, Zhao Y. KLF4 suppresses HDACi induced caspase activation and the SAPK pathway by targeting p57(Kip2). Apoptosis. 2009;14(9):1095-1107.

36. Wang Y, van Boxel-Dezaire AH, Cheon H, Yang J, Stark GR. STAT3 activation in response to IL-6 is prolonged by the binding of IL-6 receptor to EGF receptor. Proc Natl Acad Sci USA. 2013;110(42):16975-16980.

37. Kunisada K, et al. Activation of JAK-STAT and MAP kinases by leukemia inhibitory factor through gp 130 in cardiac myocytes. Circulation. 1996;94(10):2626-2632.

38. Ju W, et al. Defining cell-type specificity at the transcriptional level in human disease. Genome Res. 2013;23(11):1862-1873.

39. Arakawa T, et al. Activation of signal transducer and activator of transcription 3 correlates with cell proliferation and renal injury 
in human glomerulonephritis. Nephrol Dial Transplant. 2008;23(11):3418-3426.

40. Nagata M, Nakayama K, Terada Y, Hoshi S, Watanabe T. Cell cycle regulation and differentiation in the human podocyte lineage. Am J Pathol. 1998;153(5):1511-1520.

41. Shankland SJ, Eitner F, Hudkins KL, Goodpaster T, D'Agati V, Alpers CE. Differential expression of cyclin-dependent kinase inhibitors in human glomerular disease: role in podocyte proliferation and maturation. Kidney Int. 2000;58(2):674-683.

42. Hiromura K, et al. Podocyte expression of the CDK-inhibitor p57 during development and disease. Kidney Int. 2001;60(6):2235-2246. 43. Petermann AT, et al. Mitotic cell cycle proteins increase in podocytes despite lack of proliferation. Kidney Int. 2003;63(1):113-122.

44. Barisoni L, Mokrzycki M, Sablay L, Nagata M, Yamase H, Mundel P. Podocyte cell cycle regulation and proliferation in collapsing glomerulopathies. Kidney Int. 2000;58(1):137-143.

45. Hagen M, et al. Cell cycle re-entry sensitizes podocytes to injury induced death. Cell Cycle. 2016;15(14):1929-1937.

46. Liapis H, Romagnani P, Anders HJ. New insights into the pathology of podocyte loss: mitotic catastrophe. Am J Pathol. 2013;183(5):1364-1374.

47. Gu L, et al. Deletion of podocyte STAT3 mitigates the entire spectrum of HIV-1-associated nephropathy. AIDS. 2013;27(7):1091-1098.

48. Zhang H, et al. Podocyte-specific JAK2 overexpression worsens diabetic kidney disease in mice. Kidney Int. 2017;92(4):909-921.

49. Moreira D, et al. TLR9 signaling through NF-kB/RELA and STAT3 promotes tumor-propagating potential of prostate cancer cells. Oncotarget. 2015;6(19):17302-17313.

50. Zhang L, et al. Paclitaxel attenuates renal interstitial fibroblast activation and interstitial fibrosis by inhibiting STAT3 signaling Drug Des Devel Ther. 2015;9:2139-2148.

51. Matsui F, Babitz SA, Rhee A, Hile KL, Zhang H, Meldrum KK. Mesenchymal stem cells protect against obstruction-induced renal fibrosis by decreasing STAT3 activation and STAT3-dependent MMP-9 production. Am J Physiol Renal Physiol. 2017;312(1):F25-F32

52. Edwards LJ, Mizui M, Kyttaris V. Signal transducer and activator of transcription (STAT) 3 inhibition delays the onset of lupus nephritis in MRL/1pr mice. Clin Immunol. 2015;158(2):221-230.

53. Takemoto S, et al. Proliferation of adult T cell leukemia/lymphoma cells is associated with the constitutive activation of JAK/ STAT proteins. Proc Natl Acad Sci USA. 1997;94(25):13897-13902.

54. Kritikou EA, et al. A dual, non-redundant, role for LIF as a regulator of development and STAT3-mediated cell death in mammary gland. Development. 2003;130(15):3459-3468.

55. Heinrich PC, Behrmann I, Müller-Newen G, Schaper F, Graeve L. Interleukin-6-type cytokine signalling through the gp130/ Jak/STAT pathway. Biochem J. 1998;334 ( Pt 2):297-314.

56. Lee SJ, Borsting E, Declèves AE, Singh P, Cunard R. Podocytes express IL-6 and lipocalin 2/ neutrophil gelatinase-associated lipocalin in lipopolysaccharide-induced acute glomerular injury. Nephron Exp Nephrol. 2012;121(3-4):e86-e96.

57. Kuravi SJ, et al. Podocytes regulate neutrophil recruitment by glomerular endothelial cells via IL-6-mediated crosstalk. $J$ Immunol. 2014;193(1):234-243.

58. Braun GS, et al. IL-6 Trans-Signaling Drives Murine Crescentic GN. J Am Soc Nephrol. 2016;27(1):132-142.

59. Mallipattu SK, et al. Krüppel-Like Factor 15 Mediates Glucocorticoid-Induced Restoration of Podocyte Differentiation Markers. J Am Soc Nephrol. 2017;28(1):166-184.

60. Katz JP, et al. The zinc-finger transcription factor Klf4 is required for terminal differentiation of goblet cells in the colon. Development. 2002;129(11):2619-2628.

61. Mallipattu SK, et al. Krüppel-like factor 6 regulates mitochondrial function in the kidney. J Clin Invest. 2015;125(3):1347-1361

62. Park JW, Han CR, Zhao L, Willingham MC, Cheng SY. Inhibition of STAT3 activity delays obesity-induced thyroid carcinogenesis in a mouse model. Endocr Relat Cancer. 2016;23(1):53-63.

63. Park JW, Zhao L, Willingham MC, Cheng SY. Inhibition of STAT3 signaling blocks obesity-induced mammary hyperplasia in a mouse model. Am J Cancer Res. 2017;7(3):727-739.

64. Takemoto M, et al. A new method for large scale isolation of kidney glomeruli from mice. Am J Pathol. 2002;161(3):799-805.

65. Katsuya K, Yaoita E, Yoshida Y, Yamamoto Y, Yamamoto T. An improved method for primary culture of rat podocytes. Kidney Int. 2006;69(11):2101-2106.

66. Ohse T, Pippin JW, Vaughan MR, Brinkkoetter PT, Krofft RD, Shankland SJ. Establishment of conditionally immortalized mouse glomerular parietal epithelial cells in culture. J Am Soc Nephrol. 2008;19(10):1879-1890.

67. Dai Y, et al. Retinoic acid improves nephrotoxic serum-induced glomerulonephritis through activation of podocyte retinoic acid receptor $\alpha$. Kidney Int. 2017;92(6):1444-1457. 